\title{
Submillimeter galaxies behind the Bullet cluster (1E 0657-56)
}

\author{
D. Johansson ${ }^{1}$, C. Horellou ${ }^{1}$, M. W. Sommer ${ }^{2}$, K. Basu ${ }^{3,2}$, F. Bertoldi ${ }^{2}$, M. Birkinshaw ${ }^{4}$, K. Lancaster ${ }^{4}$, \\ O. Lopez-Cruz ${ }^{5}$, and H. Quintana ${ }^{6}$
}

1 Onsala Space Observatory, Chalmers University of Technology, 43992 Onsala, Sweden e-mail: daniel.p.johansson@chalmers.se

2 Argelander-Institut für Astronomie, Auf dem Hügel 71, 53121 Bonn, Germany

3 Max-Planck Institut für Radioastronomie, Auf dem Hügel 69, 53121 Bonn, Germany

4 Department of Physics, University of Bristol, Tyndall Avenue, Bristol BS8 1TL, UK

5 Instituto Nacional de Astrofísica, Optica y Electrónica (INAOE), Tonantzintla, Puebla 72840, Mexico

6 Departamento de Astronomía y Astrofísica, Pontificia Universidad Católica de Chile, Casilla 306, Santiago 22, Chile

Received 9 December 2009 / Accepted 19 February 2010

\begin{abstract}
Context. Clusters of galaxies are effective gravitational lenses able to magnify background galaxies and making it possible to probe the fainter part of the galaxy population. Submillimeter galaxies, which are believed to be star-forming galaxies at typical redshifts of 2 to 3, are a major contaminant to the extended Sunyaev-Zeldovich (SZ) signal of galaxy clusters. For a proper quantification of the SZ signal the contribution of submillimeter galaxies needs to be quantified.

Aims. The aims of this study are to identify submillimeter sources in the field of the Bullet cluster (1E 0657-56), a massive cluster of galaxies at $z \simeq 0.3$, measure their flux densities at $870 \mu \mathrm{m}$, and search for counterparts at other wavelengths to constrain their properties.

Methods. We carried out deep observations of the submillimeter continuum emission at $870 \mu \mathrm{m}$ using the Large APEX BOlometer CAmera (LABOCA) on the Atacama Pathfinder EXperiment (APEX) telescope. Several numerical techniques were used to quantify the noise properties of the data and extract sources.

Results. In total, seventeen sources were found. Thirteen of them lie in the central 10 arcmin of the map, which has a pixel sensitivity of $1.2 \mathrm{mJy}$ per $22^{\prime \prime}$ beam. After correction for flux boosting and gravitational lensing, the number counts are consistent with published submm measurements. Nine of the sources have infrared counterparts in Spitzer maps. The strongest submm detection coincides with a source previously reported at other wavelengths, at an estimated redshift $z \simeq 2.7$. If the submm flux arises from two images of a galaxy magnified by a total factor of 75 , as models have suggested, its intrinsic flux would be around $0.6 \mathrm{mJy}$, consistent with an intrinsic luminosity below $10^{12} L_{\odot}$.
\end{abstract}

Key words. galaxies: individual: MMJ065837-5557.0 - galaxies: clusters: individual: 1E 0657-56 - submillimeter: galaxies infrared: galaxies - cosmology: observations

\section{Introduction}

The large concentrations of mass (up to $10^{15} M_{\odot}$ ) on angular scales of a few arcminutes in galaxy clusters act as natural gravitational lenses capable of magnifying background galaxies that would be too dim to be detectable otherwise. In the $\mathrm{mm}$ and submm wavebands, lensing by galaxy clusters makes it possible to probe the fainter part of the brightness distribution of the socalled submillimeter galaxies (SMGs), which are believed to be dusty high-redshift star-forming galaxies (Blain 1997).

Pioneering observations of SMGs at 450 and $850 \mu \mathrm{m}$ were done using SCUBA on the James Clerk Maxwell Telescope. The first observations toward two massive clusters at $z \simeq 0.35$ resulted in the detection of a total of six sources above the noise level of $2 \mathrm{mJy} / \mathrm{beam}$ at $850 \mu \mathrm{m}$ (Smail et al. 1997). The authors estimated the surface density of the sources to be three orders of magnitude larger than the expectation from a nonevolving model using the local IRAS $60 \mu \mathrm{m}$ luminosity function. Those observations provided evidence for the presence of a large number of actively star-forming galaxies at high redshift, which might be the counterparts of the luminous and ultraluminous infrared galaxies observed in the local universe (e.g. Sanders \& Mirabel 1996).
At redshifts beyond one, the flux density of a redshifted infrared-luminous galaxy is largely redshift-independent: its decrease with an increasing distance is compensated by the steep rise in the $\mathrm{mm}$ and submm due to the redshifted spectral energy distribution (Blain \& Longair 1993). During the last decade, several hundreds of SMGs have been discovered using bolometer arrays, mostly SCUBA (e.g. Blain 1998; Borys et al. 2003; Coppin et al. 2006), and more recently MAMBO at $1.2 \mathrm{~mm}$ and AzTEC at $1.1 \mathrm{~mm}$ (Bertoldi et al. 2007; Scott et al. 2008; Austermann et al. 2010). The $\mathrm{mm} / \mathrm{submm}$ galaxy population is the subject of many multi-wavelength studies (see the review by Blain et al. 2002). The median redshift of SMGs with known redshifts is around 2-3 (Smail et al. 2000).

So far, only a handful of massive galaxy clusters have been mapped in the submm, and most of them are clusters in the northern hemisphere observed with SCUBA. Observation of seven massive clusters with a sensitivity of $2 \mathrm{mJy} / \mathrm{beam}$ provided a catalogue of 17 submm sources brighter than the $50 \%$ completeness limit (Smail et al. 1998). Nine other cluster fields in the redshift range $0.2-0.8$ were observed with a similar sensitivity, resulting in the detection of 17 new submm sources (Chapman et al. 2002). Deeper observations with a 3-sigma limit of $1.5-2 \mathrm{mJy} / \mathrm{beam}$ of three massive clusters probed the sub-mJy 
number counts, because of the gravitational magnification of the clusters. (Cowie et al. 2002). Knudsen et al. (2008) targeted twelve clusters and the New Technology Telescope Deep Field. They detected 59 sources (some of them being multiple images of the same galaxy), and determined that seven of them have sub-mJy lensing-corrected flux densities.

The LABOCA bolometer camera on APEX has been used to survey the $870 \mu \mathrm{m}$ emission in a protocluster at $z \simeq 2.4$ (Beelen et al. 2008) and the Extended Chandra Deep Field South Weiß et al. (2009). Nord et al. (2009) observed the Sunyaev-Zeldovich (SZ) increment toward the massive cluster Abell 2163 and noted one bright point source. This is a good candidate for an SMG lensed by the cluster.

The Bullet cluster $(1 \mathrm{E} \mathrm{0657-56)}$ at $z \simeq 0.3$ is one of the most massive galaxy clusters known to date (see Markevitch et al. 2002; Springel \& Farrar 2007). A bright millimeter source was recently discovered in the Bullet cluster field and identified as the lensed image of a background galaxy at a redshift of about 2.7 (Wilson et al. 2008, hereafter W08). The source happens to lie close to a critical line of the lens, causing a large flux amplification. The observations were performed with the AzTEC bolometer camera on the 10-m ASTE telescope in the Atacama desert in Chile, which provides an angular resolution of $30^{\prime \prime}$ at $1.1 \mathrm{~mm}$ wavelength. A doubly lensed source at the same location had been previously identified in Hubble Space Telescope (HST) maps and was used, together with other multiply lensed galaxies and a large number of weakly lensed sources, to obtain a calibrated map of the projected mass distribution of the Bullet cluster (Bradač et al. 2006). Recently, Gonzalez et al. (2009) identified a third image by analyzing maps in the optical (HST), and in the near- and mid-infrared (Magellan and Spitzer). By fitting the spectral energy distribution (SED) of a starburst galaxy to the observations, they also inferred a redshift of about 2.7. Their lensing model gave a magnification of 10-50 for the three images.

In this paper we present results of observations of the Bullet cluster field at a wavelength of $870 \mu \mathrm{m}$ using the LABOCA bolometer camera. At that wavelength, the emission is a combination of extended signal due to the SunyaevZeldovich effect by the hot intracluster gas and of point sources, which are potential high-redshift star-forming galaxies. Recovery of the extended SZ signal from the LABOCA data (the SZ increment) requires a different data reduction and will be presented in a subsequent paper. The SZ decrement from the Bullet cluster has been mapped by e.g. Halverson et al. (2009) with the APEX-SZ instrument, operating at $2 \mathrm{~mm}$. This paper is organized as follows: the observations are presented in Sect. 2.1 and the data reduction in Sect. 3; the results are presented and discussed in Sects. 4 and 5.

Throughout the paper, we adopt the following cosmological parameters: a Hubble constant $H_{0}=70 \mathrm{~km} \mathrm{~s}^{-1} \mathrm{Mpc}^{-1}$, a matter density parameter $\Omega_{0}=0.3$, and a dark energy density parameter $\Omega_{\Lambda 0}=0.7$. The redshift $z=0.296$ of the Bullet cluster corresponds to an angular-diameter distance of $910 \mathrm{Mpc}$ and a scale of $4.41 \mathrm{kpc} / \mathrm{arcsec}$.

\section{Observations}

\subsection{Submillimeter}

The observations ${ }^{1}$ were carried out in September, October and November 2007 using LABOCA (Large APEX BOlometer

\footnotetext{
${ }^{1}$ Swedish program ID O-079.F-9304A and ESO program ID E-380.A-3036A.
}

CAmera, Siringo et al. 2009) on the APEX telescope ${ }^{2}$ (Güsten et al. 2006). LABOCA is a 295-element receiver operating at a central frequency of $345 \mathrm{GHz}$ with a bandwidth of $60 \mathrm{GHz}$. At the time of our observations, about 250 bolometers were used. The mean point-source sensitivity of those bolometers was $78 \mathrm{mJy} \mathrm{s}^{1 / 2}$. The angular resolution was $19.5^{\prime \prime}$ and the field-ofview was $11.4^{\prime}$. The layout of the bolometer array is illustrated in Fig. 1. A total of $25 \mathrm{~h}$ of observing time was spent, including pointing and calibration. The weather conditions were varying, with an amount of precipitable water vapor ranging from 0.5 to $2.0 \mathrm{~mm}$.

LABOCA uses feed horn antennas and their physical size limits the spacing of the bolometers on the array. LABOCA is therefore not fully sampling the sky, so the telescope has to be moved to sample the sky such that the resulting map meets the Nyquist criterion. The scanning pattern does not only "fill the gaps" between bolometers, it also modulates the astronomical signal into a range of spatial frequencies which facilitates filtering of $1 / f$-type noise (instrumental and sky noise).

\subsubsection{Scanning patterns}

We used two different scanning patterns, as illustrated in Fig. 1. Both are a combination of Archimedian spirals with a duration of $35 \mathrm{~s}$, centered on a four-point raster.

- During the first observing session, we used a compact scanning pattern: the four points were separated by $27^{\prime \prime}$ in azimuth and elevation, each point marking the center of a spiral with a minimum radius of $R_{0}=18^{\prime \prime}$ and winding out with a radial speed of $\dot{R}=2.25^{\prime \prime} \mathrm{s}^{-1}$ and an angular speed of $\dot{\phi}=90 \mathrm{deg} \mathrm{s}^{-1}$. The spirals thus ranged from $18^{\prime \prime}$ to about $97^{\prime \prime}$ in radius, with a scanning speed between $0.5^{\prime} \mathrm{s}^{-1}$ and $2.5^{\prime} \mathrm{s}^{-1}$.

- During the second observing session, a larger scanning pattern was used to facilitate the retrieval of the extended SZ signal. The four raster points were separated by $100^{\prime \prime}$ in azimuth and elevation, and the spirals had a minimum radius $R_{0}=120^{\prime \prime}$ and radial and angular speeds of $\dot{R}=1.25^{\prime \prime} \mathrm{s}^{-1}$, $\dot{\phi}=90 \mathrm{deg} \mathrm{\textrm {s } ^ { - 1 }}$. The spirals thus ranged from 120 to $164^{\prime \prime}$ in radius, with a scanning speed between 3 and $4^{\prime} \mathrm{s}^{-1}$.

\subsubsection{Pointing, focus and calibration}

The pointing accuracy was checked by repeated observations of the nearby source PKS 0537-441. This is a variable source; during our observations, its mean flux density was $\sim 3 \mathrm{Jy}$. The source was scanned in a tight spiral and the data were reduced and made into a map using the BoA software (see Sect. 3). A two-dimensional Gaussian was fitted to the pointing source, and the telescope's pointing was updated using offsets from the fit. The pointing was stable within $3^{\prime \prime}$.

The focus was checked at least twice during every observing session by observing a planet (Venus, Saturn or Mars). The subreflector was moved in small increments in each of the three cartesian directions while the telescope tracked the source. The optimum focus position in each direction was determined by fitting a curve to the observed points, and the subreflector was finally moved to the position corresponding to the maximum of the curve.

2 This publication is based on data acquired with the Atacama
Pathfinder EXperiment (APEX). APEX is a collaboration between
the Max-Planck-Institut für Radioastronomie, the European Southern Observatory, and Onsala Space Observatory. 

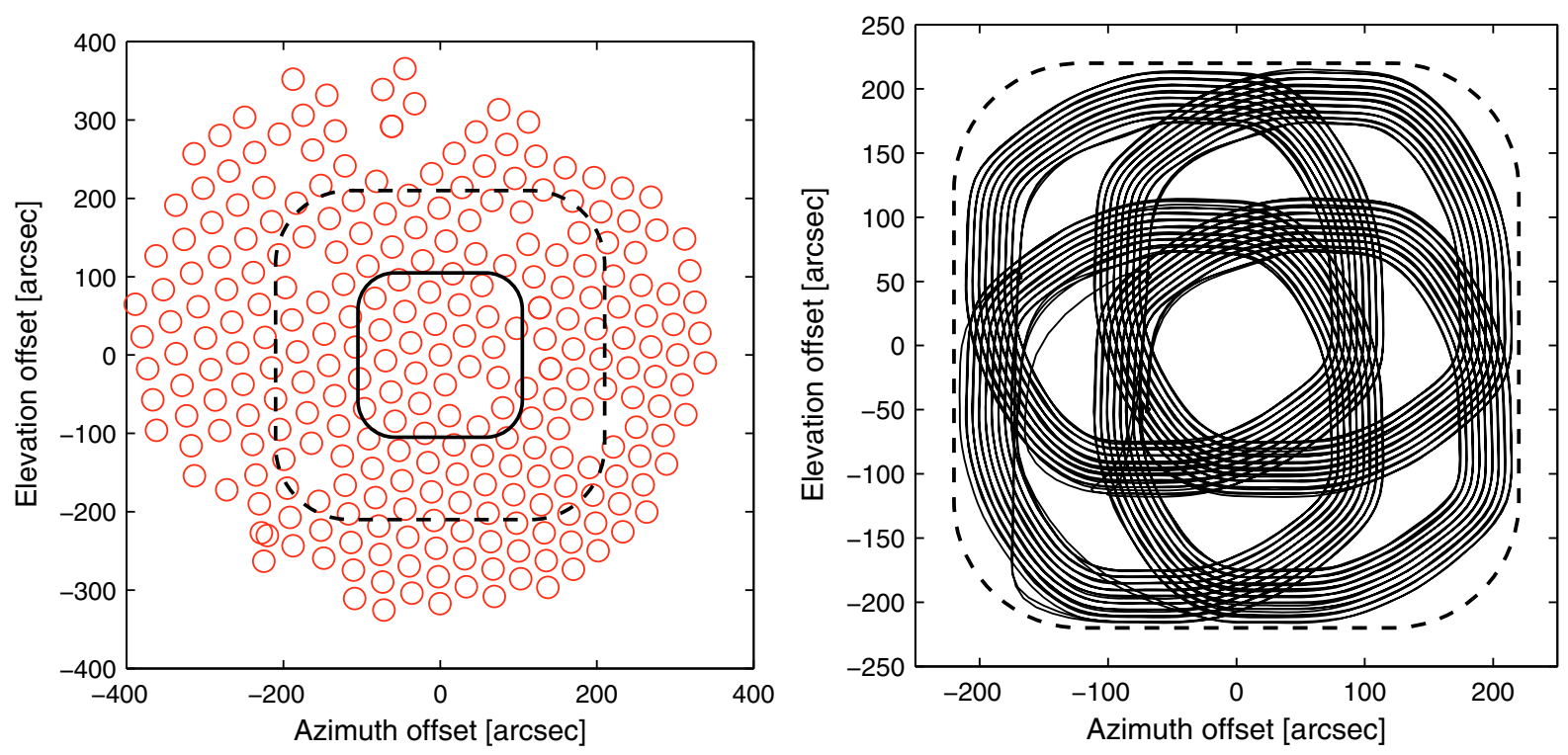

Fig. 1. Left: layout of the bolometers on the LABOCA array. Each circle represents one bolometer that was active at the time of the observations. The two rectangles illustrate the sizes of the two raster+spiral scanning pattern for one bolometer (solid line-small pattern, dashed line-large pattern). Right: movement pattern for one bolometer during the large scanning pattern. This pattern has a $2 \times 2$ raster setup with $100^{\prime \prime}$ between the raster points (the patterns are described in detail in Sect. 2.1). The smaller scanning pattern is similar, but because the distance between raster points is considerably smaller, it is harder to illustrate. A comparison between the two patterns can instead be made from the two rectangles in the left panel. For clarity, we plot also the dashed rounded rectangle of the left panel in the right panel.

The absolute flux calibration of LABOCA is supposed to be accurate to $10 \%$ (Siringo et al. 2009). We verified this by daily observations of Uranus.

The atmospheric attenuation was determined from continuous scans in elevation at a fixed azimuth ("skydips"), and from radiometer measurements (Siringo et al. 2009).

\subsection{Infrared}

In Sect. 5.1 we describe a search for infrared counterparts to the detected submm sources in Spitzer maps. We now describe the data that was used for that comparison.

Spitzer IRAC and MIPS of the Bullet cluster field were acquired from the Spitzer data archive. Both the IRAC and MIPS data were taken under program ID 40593 (PI: Gonzalez). The Spitzer data overlap with most of the the region observed with LABOCA, and out of the detected submm sources only one source lacks Spitzer coverage.

The IRAC and MIPS maps were processed by, respectively, version 18.7 and 18.12 of the SSC pipeline. We started by visually inspecting the resulting pbcd (post basic calibrated data) mosaics, and found that the IRAC maps had no apparent artefacts, but that the MIPS map had clear signs of "dark latents", as described in the MIPS data handbook. We therefore reprocessed the basic calibrated data (bcd) using MOPEX version 18.3.3 the script flatfield.pl to self-calibrate the bcd data. The bcd's were then mosaiced using mopex.pl. The reprocessed MIPS map shows no signs of artefacts due to "latents".

Properties of the acquired Spitzer data are summarized in Table 1. We list there the median integration time per pixel per map, the sensitivity and the angular size of each map. The sensitivity is estimated from the final combined mosaics, masking out all pixels brighter than 10 times the median pixel value in each map, and then calculating the standard deviation of the remaining pixels. The listed sensitivity values are $3 \sigma$, and are similar
Table 1. Properties of the Spitzer data used in this study.

\begin{tabular}{lccc}
\hline \hline Band & $\begin{array}{c}t_{\text {int }} \\
(\mathrm{s})\end{array}$ & $\begin{array}{c}3 \sigma \text {-depth } \\
(\mu \mathrm{Jy})\end{array}$ & $\begin{array}{c}\text { FOV } \\
(\operatorname{arcmin} \times \text { arcmin })\end{array}$ \\
\hline IRAC1 & 9100 & 0.9 & $15 \times 22$ \\
IRAC2 & 9100 & 1.4 & $15 \times 22$ \\
IRAC3 & 9100 & 6.6 & $15 \times 22$ \\
IRAC4 & 9100 & 7.0 & $15 \times 22$ \\
MIPS & 7800 & 39.5 & $13.4 \times 12.7$ \\
\hline
\end{tabular}

to those obtained from the Spitzer Science Center "Sensitivity Performance Estimation Tool”3.

\section{Data reduction}

In this section, we describe the steps followed to produce a fully calibrated map from the raw data, which come in the form of time-streams containing the voltage read-outs of each bolometer as a function of time. We have used two data reduction softwares: Minicrush ${ }^{4}$, written originally for the SHARC bolometer array and adapted to handle LABOCA data (Kovács 2008), and $B o A^{5}$, developed in Bonn (Schuller et al., in prep). In general, the maps produced by both pipelines were in good agreement in terms of number and characteristics of sources; however, since the Minicrush map showed a lower level of large-scale noise, we used that software for the analysis presented in this paper.

The data consist of a total of 185 eight-minute-long scans on the cluster, plus pointing and calibration observations. Each scan is contained in a separate MBFITS-file.

\footnotetext{
3 http://ssc.spitzer.caltech.edu/tools/senspet/

4 Minicrush can be downloaded from http://www. submm. caltech.edu/ sharc/crush/download.htm.

5 BoA can be downloaded from http://www.apex-telescope. org/bolometer/laboca/boa/.
} 
First, we flagged blind bolometers to exclude them from the rest of the data analysis. Then, we corrected for differences in sensitivity of individual bolometers. That information was extracted from "beam-maps", which are fully sampled maps where each bolometer has scanned a bright planet.

For each scan, the zenith opacity calculated from the radiometer and skydip measurements was used to calibrate the data for elevation-dependent opacity variations. We also flagged data taken during periods of high telescope speeds and accelerations.

\subsection{Removal of correlated noise in the time-streams}

The most critical and challenging task of the data analysis is to extract the true astronomical signal from the measurements, which are contaminated by noise from various sources. One component of the noise arises in the electronic systems, such as the readouts of the bolometer array; its spectrum is of the $1 / f$ type where $f$ is the frequency. Even more important is the contribution of the Earth's atmosphere, which also has a $1 / f$-type spectrum, but shows both spatial and temporal fluctuations. In the $870 \mu \mathrm{m}$ atmospheric window in which LABOCA operates, typical zenith opacities at the APEX site are of the order of 0.1 to 0.2 . The atmosphere is thus largely transparent to cosmic signals; but the amplitude of the atmospheric signal can be as high as $10^{5}$ times that of the astronomical signal of interest. Because the atmospheric signal (or noise) is correlated across the bolometer array, it can be estimated and removed from the time-streams. For point-source observations, correlated sky noise can be partly removed by filtering low spatial frequencies (or large angular scales), where the $1 / f$ noise is most severe.

The software Minicrush takes the following approach to remove correlated noise from the time-streams: a correlated noise component is modeled as a common signal in the timestreams of the different bolometers, scaled by a gain factor which depends on each bolometer. A $\chi^{2}$ function is minimized by taking its derivative with respect to the modeled signal. The time-streams of all the bolometers are considered for a certain scan. When the fit has been performed, the estimated correlated signal is removed from the data. The process of estimation and removal of the correlated signal is carried out several times. The uncertainties on the modeled correlated signal are estimated by calculating the changes in the estimated signal when the $\chi^{2}$ has increased by 1 from its minimum value. The ideas implemented in Minicrush have been described in detail in Kovács (2008).

Since we were interested in compact sources, we used the option-deep in Minicrush.

\subsection{Map-making}

\subsubsection{From time-streams to maps}

The data consist of a collection of time-streams from each functioning bolometer, cleaned from atmospheric and instrumental noise. The data in each time-stream of a scan are used to create a map of that scan, taking into account the scanning pattern of the telescope and using a nearest-pixel mapping algorithm. We refer to those maps as scan-maps. For each scan-map, a corresponding noise map is generated by adding in quadrature the noise levels of the bolometers that hit a certain pixel in the map. The noise maps are used to weigh the individual scan maps when they are co-added, which is the final step of the map-making process. The individual noise maps are also coadded into a final noise map. This noise map reflects the pixel $\mathrm{rms}$ in the data. As the noise map only accounts for the relative weights between map pixel,

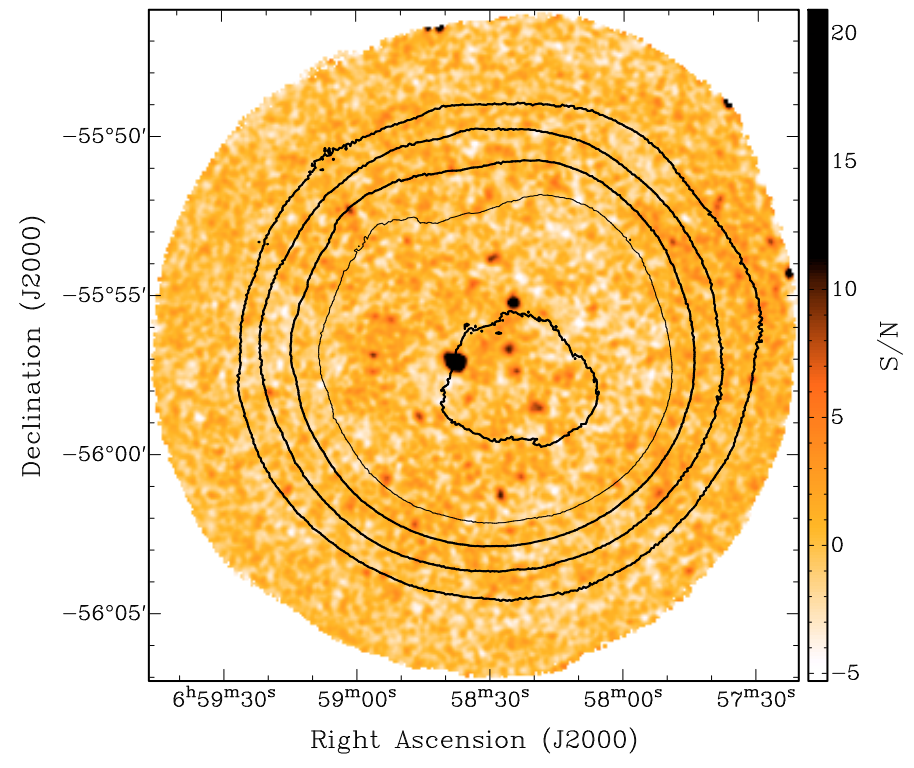

Fig. 2. Color image of the $870 \mu \mathrm{m}$ signal-to-noise map. The map has been filtered to remove extended signal, including that due to the Sunyaev-Zeldovich effect. Several sources are visible. The brightest coincides with the millimeter source discovered by W08 and identified as a galaxy at redshift $z \simeq 2.7$ and strongly lensed by the Bullet cluster. The contours refer to the noise level at 1.3, 2.1, 2.7, 4.1 and $6.8 \mathrm{mJy} / \mathrm{beam}$. The signal-to-noise representation supresses the high noise levels in the outer parts of the map. They are instead indicated in the contours of the noise map.

we then rescaled the noise map. In Sect. 4.1 we show that the signal-to-noise map has a Gaussian distribution of pixel values. We scaled the noise map in order for the signal-to-noise map to have a pixel histogram with a standard deviation of 1 . This process scales the noise map with a factor of 1.5. The rescaled noise map is shown as contours in Fig. 2, together with the the signal-to-noise map which is constructed by dividing the signal map with the scaled noise map.

The maps have a pixel size of $4^{\prime \prime}$. This means that the oversampling factor compared with the original resolution of the observations is $19.5 / 4 \sim 5$. Such a fine pixelization is preferential for the map-making process to be effective. The resulting map has a pixel-to-pixel noise level which is affected by high frequency noise on a scale smaller than the beam. This noise (which is due to small pixelization) can easily be removed by smoothing. We smoothed the final maps using a 10" Gaussian in order to remove that high-frequency noise component and produce cleaner maps.

\subsubsection{Iterative mapping}

Some artefacts such as "sidelobes" around point sources are seen in the final map because of the filtering. In order to remove those artefacts, the entire Minicrush reduction was applied a second time, but instead of building a source model from the actual data being reduced, we used a source model based on the results of the first reduction. The part of the map with a signal above $4.5 \sigma$ was used as the source model.

Figure 3 illustrates the result of that iterative process: radial profiles of the brightest source in the map are shown for each of the two iterations. The difference between the two profiles is significant. We have observed that the difference is greater the brighter the source. The iterative process makes it possible to 


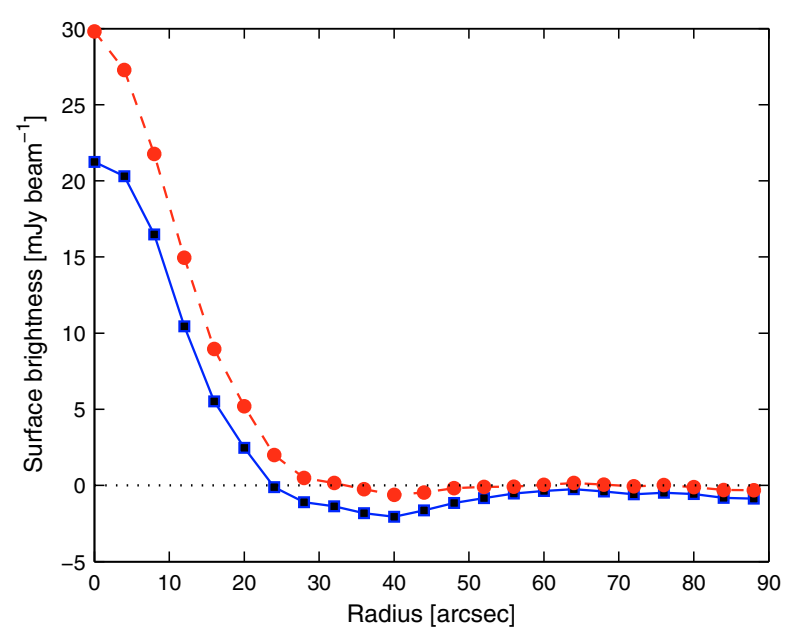

Fig. 3. Radial profiles of the brightest source (source \#1): in the first iteration of the data reduction (solid line and boxes), a source model was successively built during the data reduction; in the second iteration (dashed line + circles), a $4.5 \sigma$-clipped version of the first map was used as a source model. The iterative method makes it possible to recover flux that was lost at the first stage of the data reduction.

recover the flux that was lost in the first iteration. We have compared the fluxes of our two most significant sources (after two iterations) with the values obtained using the standard data reduction pipeline that was used for the calibrator, Uranus. The agreement showed that two iterations were sufficient, as also found by other groups (e.g. Weiß et al. 2009).

\section{Results}

Figure 2 shows the signal-to-noise map obtained from the data reduction process described above. Several sources are visible. Since the noise level increases steeply toward the outer parts, sources with low signal-to-noise ratio in that part of the map may have high flux densities. In this section, we discuss the noise properties of the data and present the methods that we have used to identify sources and measure their properties. We also present Monte Carlo simulations performed to quantify the degree of completeness to which sources can be extracted, and to estimate the amount of flux boosting due to the confusion noise. Finally, we estimate the magnification of each source due to gravitational lensing, using a simple model of the Bullet cluster and assuming that all sources are at a redshift of 2.5.

As shown in the following analysis, neither completeness nor flux boosting corrections change the results very much, because of the conservative detection treshold that we have adopted. The result is a fairly robust catalog of 17 sources.

\subsection{Noise properties}

To quantify the noise level in the map, we constructed 500 so-called jackknife noise maps, which are obtained by multiplying half of scan maps (selected randomly) by (-1), before co-adding all the scan maps. The jackknife maps should therefore be free from astronomical sources and from artefacts from the data reduction pipeline, and reveal the nature of the statistical noise in the data. Each jackknife map was smoothed with a $10^{\prime \prime}$ Gaussian after coadding, giving a final resolution of $\sqrt{\left(19.5^{\prime \prime}\right)^{2}+\left(10^{\prime \prime}\right)^{2}} \simeq 22^{\prime \prime}$. Then, we calculated the mean

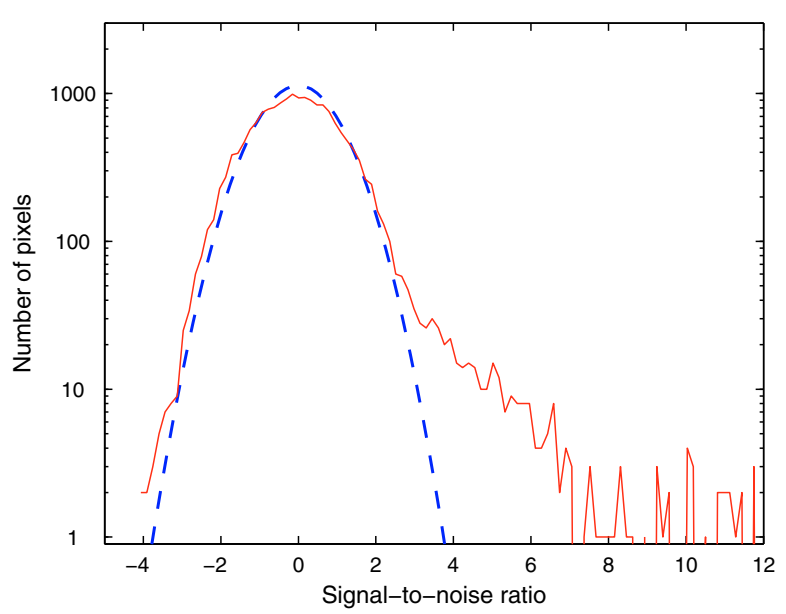

Fig. 4. Solid curve: histogram of the pixel distribution in the central 10 arcmin of the signal-to-noise map. Dashed curve: histogram of the mean pixel distribution in the same region of the jackknife maps, as described in the text. The jackknife procedure has removed the excess positive signal (due to the sources) and the negative signal (due to the sidelobes) that are present in the signal map.

of the 500 histograms of the pixel distributions measured in the central $10^{\prime}$ of each jackknife map.

Figure 4 shows the mean histogram, in units of signal-tonoise value, together with the histogram of the pixel distribution of the signal-to-noise map, extracted from the same central $10^{\prime}$. The jackknife histogram is fitted by a Gaussian with standard deviation 1 (as expected). If we instead make the same calculation in the signal map, we find that the jackknife histogram is well fitted by a Gaussian function with mean $\mu=1.4 \times 10^{-6} \mathrm{mJy}$ and standard deviation $\sigma=1.17 \mathrm{mJy}$. Therefore, when excluding astronomical sources and systematic effects from the data reduction, the statistical noise level is $1.2 \mathrm{mJy} / \mathrm{beam}$.

\subsection{Source extraction}

Although the noise in our final map is fairly uniform across the central 10 arcmin, it increases slightly with radius across that area, and rapidly outside (see the noise map contours in Fig. 3). In order to identify significant sources in the map, a well-defined and mathematically justified algorithm must be used. Following other authors (e.g. Beelen et al. 2008), we used the so-called "Gaussian matched filter" (GMF) technique outlined by Serjeant et al. (2003). This method is optimal for point source extraction in a $\chi^{2}$ sense, although the performance is degraded for crowded maps (Serjeant et al. 2003).

The GMF significance map, $F / \Delta F$, is computed as

$$
\frac{F}{\Delta F}=\frac{(S \cdot W) \otimes P}{\sqrt{W \otimes P^{2}}}
$$

where $S$ is the signal map, $W$ is the weight map (the reciprocal of the noise map, squared), and $P$ is a Gaussian of the same size as the beam. The $\otimes$ sign denotes a convolution.

We generated two GMF maps, one for each iteration performed in Minicrush (see Sect. 3.2.2). Setting a threshold $F / \Delta F>9$, which roughly corresponds to a signal-to-noise level of 4 , we extracted 19 sources in the first GMF map and 22 sources in the second. We decided to be conservative and not include in the final catalog the sources that had appeared in the GMF map of the iterated map. In addition, we excluded the two 


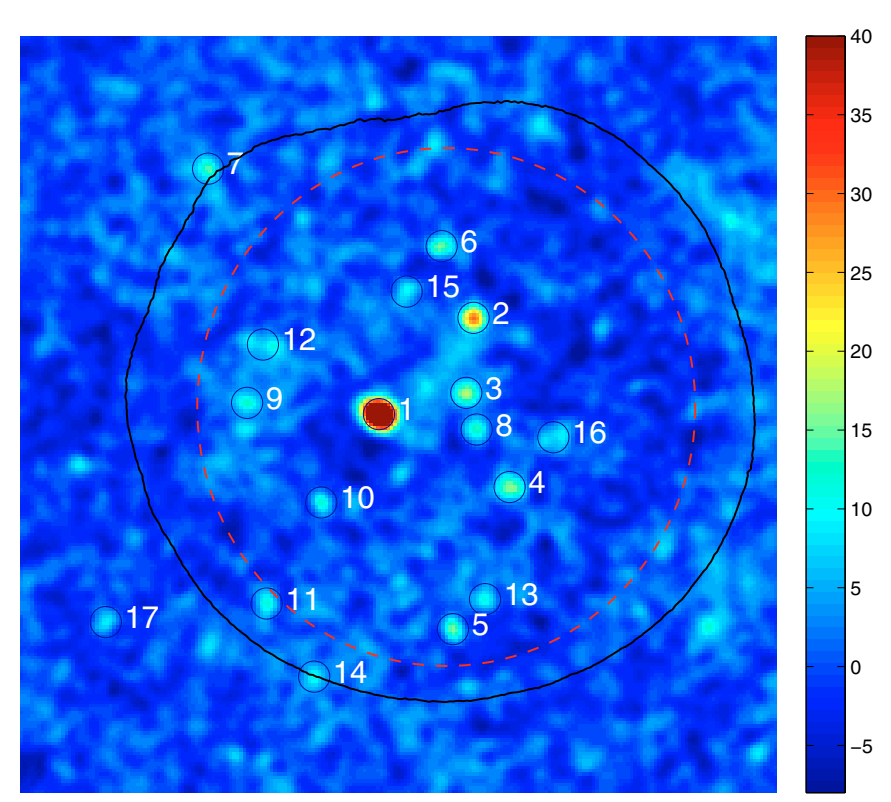

Fig. 5. Gaussian filtered map, with the 17 detected sources marked with circles. The numbering of the sources is the same as in Table 2. The black contour corresponds to the $2 \mathrm{mJy} / \mathrm{beam}$ level in the noise map and the dashed circle marks the central $10^{\prime}$ area of constant noise level used in the analysis.

sources that were present in the first GMF map but not in the second. Our final source list thus contains 17 sources.

Figure 5 shows the GMF map calculated using the iterated signal map. The black contour indicates the $2 \mathrm{mJy} / \mathrm{beam}$ noise level, and the red circle has a diameter of $10 \mathrm{arcmin}$. The $17 \mathrm{iden}-$ tified sources are marked.

Whereas source finding was done in the GMF map, measurement of the properties of the identified sources was done in the signal map. The following scheme was used:

1. We searched for the peak value in the GMF map and fitted a two-dimensional Gaussian.

2. The position obtained from that fit was used as a starting guess in the fit to the real source in the signal map. The fitted function is the sum of a two-dimensional Gaussian and a tilted plane. The use of a plane reduces the effect of remaining sidelobes around bright sources. Those are due to the filtering in the data reduction and are not completely removed by the iterative method described in Sect. 3.2.2 (Fig. 3). Unless the source is clearly extended, the width of the Gaussian was fixed to that of the beam.

3. The integrated flux density of each extracted source was calculated by integrating only the Gaussian part of the fitted function.

4. The fitted Gaussian was then subtracted from the GMF map and a new search for the peak was performed.

5. We iterated over the previous steps until the peak value in the GMF map was smaller than 9. This value was inferred from the simulations described in the next section.

Table 2 lists the properties of the 17 sources, sorted by decreasing value of $F / \Delta F$, given in the last column. The fitted positions and integrated flux densities are also given. All sources but Source \#1 are point sources. The uncertainties on the extracted flux densities are estimated from the 500 jackknife maps.

\subsection{Completeness}

In order to understand the systematics of the source extraction procedure and quantify the extent to which the values of $\Delta F / F$ correspond to real sources, we turned to simulations. A Gaussian source of the size of the beam was added at a random location within the central $10^{\prime}$-diameter region of a randomly selected jackknife map, and the Gaussian filtered map was produced, using Eq. (1). We stepped through a range of values of the flux densities of the simulated source, ranging from 1 to $15 \mathrm{mJy}$, with a spacing of $0.5 \mathrm{mJy}$. For each flux density value, 500 sources were simulated and placed at a random location, and the GMF maps were used to find the simulated sources. Once they were found, a two-dimensional circular Gaussian was fitted to the simulated signal map at the same location. For each flux density bin, we then extracted information about the completeness and the recovered flux as a function of the input flux density value.

Figure 6 shows the results. The flux boosting is calculated as the fraction of the measured flux of a source to the input flux density. At flux densities larger than $6 \mathrm{mJy}$ we see that, in the absence of confusion noise, no boosting is observed. For lower flux densities the flux boosting becomes larger than one, so that extracted sources are those that are placed on positive noise peaks, making them rise above the noise. Sources randomly placed in noise voids are not extracted.

The completeness is defined as the fraction of sources that are recovered from the simulated maps out of the 500 input sources. We see that at $6 \mathrm{mJy}$ the observations are $100 \%$ complete. At the level of $4.6 \mathrm{mJy}$, which is the flux of the most dim source in our sample, the completeness is $\sim 85 \%$. Therefore, our sample is highly complete, and completeness corrections are not very important.

Other groups use instead the final signal map when simulating the completeness (e.g. Scott et al. 2008), but we wanted to focus our attention in these simulation on the effect of statistical noise in the map. No source confusion noise is present in the jackknife maps. In the following section, we discuss the effect of confusion noise and use noise-free sky realizations with a Schechter distribution to quantify the flux boosting for an individual source extracted from the real map.

\subsection{Flux boosting due to confusion noise}

Several SMG surveys have shown that the number counts steepen towards higher flux densities (e.g. Scott et al. 2006; Coppin et al. 2006). Therefore there are many more sources at low than at high flux densities. Most of those faint sources are below the noise level of submm maps, but they influence photometric measurements of extracted sources, acting as a "sea" of sources, often referred to as "confusion noise". This effect has been discussed by e.g. Condon (1974) and Hogg \& Turner (1998). Recently, Coppin et al. (2005) discussed the effect of confusion noise on flux boosting in the SCUBA Groth strip survey, and used a Bayesian technique to "deboost" the fluxes. We employ a similar technique to estimate the amount of flux boosting for our detected sources, which we describe in Appendix A. The derived flux boosting corrections are small for most sources. The deboosted flux densities are listed in Table 2.

\subsection{Lensing correction}

We built a simple lensing model of the Bullet cluster in order to estimate the magnification of our observed submm sources. The model consists of two spherically symmetric components 
Table 2. List of sources extracted from the LABOCA map.

\begin{tabular}{lcccccc}
\hline \hline Source & $\alpha(\mathrm{J} 2000)$ & $\delta(\mathrm{J} 2000)$ & Flux density $^{a}$ & $\begin{array}{c}\text { Deboosted }^{b} \\
\text { flux density } \\
(\mathrm{mJy})\end{array}$ & $\begin{array}{c}\text { Demagnified }^{c} \\
\text { flux density } \\
(\mathrm{mJy})\end{array}$ & $F / \Delta F^{d}$ \\
\hline 1 & $(\mathrm{~h}: \mathrm{m}: \mathrm{s})$ & $\left({ }^{\circ}:^{\prime}:^{\prime \prime}\right)$ & $(\mathrm{mJy})$ & 0.64 & 82.8 \\
2 & $06: 58: 37.62$ & $-55: 57: 04.8$ & $48.6 \pm 1.3^{e}$ & $48.0 \pm 1.3$ & 8.8 & 29.9 \\
3 & $06: 58: 24.47$ & $-55: 55: 12.5$ & $15.1 \pm 1.0$ & $14.7 \pm 1.0$ & 2.2 & 17.8 \\
4 & $06: 58: 25.45$ & $-55: 56: 40.1$ & $6.9 \pm 0.9$ & $6.4 \pm 1.0$ & 4.7 & 16.2 \\
5 & $06: 58: 19.36$ & $-55: 58: 30.3$ & $8.2 \pm 0.9$ & $7.7 \pm 0.9$ & 6.3 & 15.9 \\
6 & $06: 58: 27.27$ & $-56: 01: 16.3$ & $9.0 \pm 1.3$ & $8.0 \pm 1.3$ & 6.3 & 15.4 \\
7 & $06: 58: 28.94$ & $-55: 53: 48.4$ & $9.3 \pm 1.2$ & $8.6 \pm 1.2$ & 8.4 & 14.2 \\
8 & $06: 59: 01.39$ & $-55: 52: 18.1$ & $11.9 \pm 2.1$ & $9.7 \pm 2.1$ & 1.8 & 13.1 \\
9 & $06: 58: 24.05$ & $-55: 57: 23.0$ & $5.3 \pm 0.9$ & $4.7 \pm 1.0$ & 3.3 & 12.8 \\
10 & $06: 58: 55.98$ & $-55: 56: 51.7$ & $5.4 \pm 1.2$ & $4.4 \pm 1.3$ & 3.6 & 12.0 \\
11 & $06: 58: 45.60$ & $-55: 58: 48.0$ & $6.2 \pm 1.1$ & $5.5 \pm 1.1$ & 5.2 & 11.9 \\
12 & $06: 58: 53.22$ & $-56: 00: 45.0$ & $7.8 \pm 1.5$ & $6.4 \pm 1.6$ & 3.4 & 11.2 \\
13 & $06: 58: 52.22$ & $-55: 55: 45.7$ & $5.5 \pm 1.2$ & $4.5 \pm 1.2$ & 2.9 & 11.0 \\
14 & $06: 58: 22.88$ & $-56: 00: 40.7$ & $4.8 \pm 1.2$ & $3.8 \pm 1.3$ & 3.8 & 10.8 \\
15 & $06: 58: 46.68$ & $-56: 02: 11.8$ & $7.2 \pm 1.9$ & $4.6 \pm 2.5$ & 2.5 & 10.1 \\
16 & $06: 58: 33.69$ & $-55: 54: 40.8$ & $4.6 \pm 1.1$ & $3.6 \pm 1.2$ & 1.9 & 9.2 \\
17 & $06: 58: 12.44$ & $-55: 57: 29.7$ & $4.9 \pm 1.0$ & $4.2 \pm 1.0$ & - & 9.0 \\
\hline
\end{tabular}

Notes. Statistical uncertainties on the listed positions are $1-2^{\prime \prime}$, which is smaller than the pointing uncertainty. ${ }^{(a)}$ Flux density as extracted from the map. ${ }^{(b)}$ Flux density corrected for boosting due to confusion noise. ${ }^{(c)}$ Flux density corrected for lensing. ${ }^{(d)}$ Significance of the detection in the Gaussian-matched-filtered map. ${ }^{(e)}$ Source \#1 is extended relative to the $22^{\prime \prime}$ beam: it has an apparent size of $29.2^{\prime \prime} \times 23.3^{\prime \prime}$. ${ }^{(f)}$ Source \#17 lies in the outer part of the map where the noise level is high and the method used to deboost the flux densities fails.
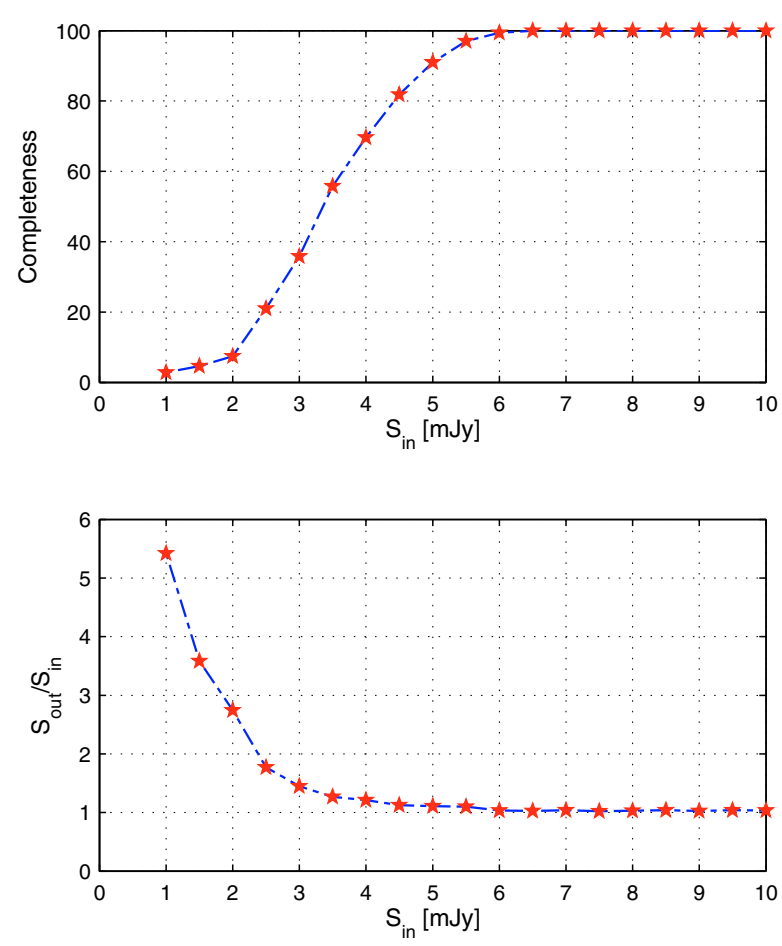

Fig. 6. Results of the Monte Carlo simulations performed to estimate the degree of completeness of the source extraction algorithm and the accuracy of the measured flux density at a given value of the input flux density.

representing the main component of the Bullet cluster and the subcomponent (the actual "bullet" to the west). Figure 7 (left) shows contours of the projected mass distribution inferred from weak-lensing observations by Clowe et al. (2006), overlaid on our $870 \mu \mathrm{m}$ image. We used the fits to the weak-lensing observations made by Bradač et al. (2006) to the masses within a certain projected radius $R$ of each component, $M(<R)=$ $M_{0}(R / 250 \mathrm{kpc})^{n}$, with $M_{0}=0.22 \times 10^{15} M_{\odot}$ and $n=0.8$ for the main cluster and $M_{0}=0.17 \times 10^{15} M_{\odot}$ and $n=1.1$ for the subcluster. To place the two mass components, we used the information given in Table 2 of the paper by Bradač et al. The redshift of our simulated Bullet cluster was set to 0.296 and that of the source plane to $z=2.5$. Note that the magnification values are not very sensitive to the redshift of the sources: varying it from $z=2$ to $z=3$ changes the magnifications by less than $10 \%$. The magnification map was calculated following the derivation in the book by Schneider et al. (1992). This numerical calculation provides results along the lines of those obtained analytically for two point masses by Schneider \& Weiss (1986) and for two isothermal spheres by Shin \& Evans (2008), but in the case of two power-law projected mass distributions. Because the model does not include lensing by the individual cluster galaxies, the location of the critical lines differ from the observed ones by about 10 arcsec. The true magnification for a given source must therefore differ from our derived values. Nevertheless, this simple model makes it possible to estimate the individual magnifications and the average magnification in a certain region. Using the calculated magnifications, we corrected the measured flux densities. The lensing-corrected values are listed in Table 2.

\section{Discussion}

\subsection{Searching for infrared counterparts to the submm sources}

The identification of optical counterparts to SMGs is often difficult due to their optical faintness, as much of the starburst luminosity is highly obscured by dusty clouds.

Rest-frame infrared photometry of SMGs is usually possible because the extinction is much smaller than in the optical. With the Spitzer satellite and its imaging photometers IRAC (Fazio et al. 2004) and MIPS (Rieke et al. 2004), it is now possible to obtain high-resolution infrared images of these distant 

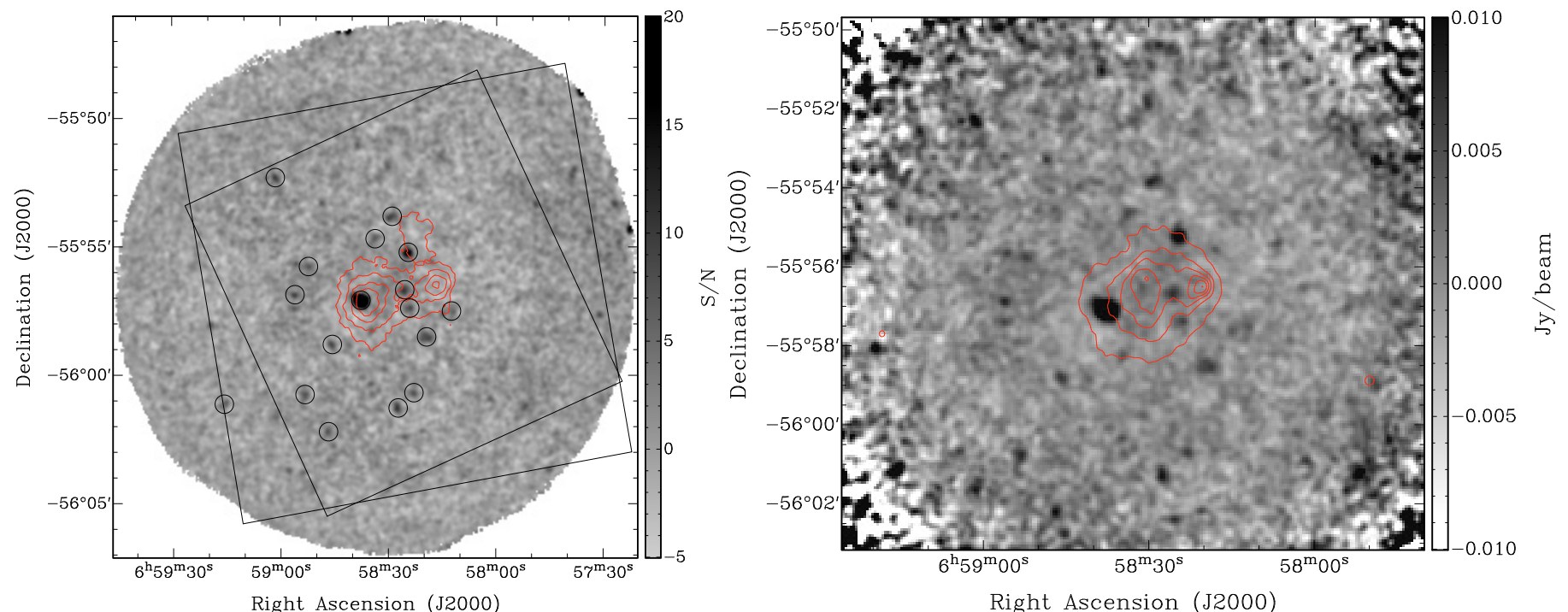

Fig. 7. Left: signal-to-noise map with overlaid circles indicating extracted submm sources. The contours show the the projected mass density from the weak lensing analysis by Clowe et al. (2006). The contours range from 40 to $85 \%$ of the maximum value and are spaced by $15 \%$. The weak lensing map was retrieved from the website http://flamingos.astro.ufl.edu/1e0657/public.html. The rectangles show the regions of complete coverage of Spitzer MIPS (small rectangle) and IRAC (large rectangle). Right: signal map (in units of Jy/beam) with contours of the $\mathrm{X}$-ray surface brightness from XMM-Newton observations. The noise level in the signal map increases rapidly towards the outskirts because of the low coverage there.

objects. The number counts of sources at $3.6 \mu \mathrm{m}$ is high and counterpart identification is complicated by the large positional uncertainty of the submm source (see below). In the $24 \mu \mathrm{m}$ band, the number density of sources is smaller and a more secure counterpart identification can be made, if a source can be identified.

Figure 8 shows cutouts of IRAC 4.5 and IRAC 8.0 and MIPS $24.0 \mu \mathrm{m}$ images of 16 sources detected in the LABOCA map. Source \#17 has only partial Spitzer coverage and is omitted from this figure. The Spitzer data used in this study are described in Sect. 2.2 and the coverage with respect to the LABOCA map is shown in Fig. 7.

The full $1^{\prime} \times 1^{\prime}$ cutouts are only shown for reference here. It is not necessary to search for infrared counterparts in such a large field. The region of interest (within a certain search radius) is the sum of the contributions from the mean pointing error of LABOCA of $\sim 4^{\prime \prime}$; the statistical uncertainties on the fitted positions, which are $1-2^{\prime \prime}$; the systematic uncertainty due to confusion noise, $\sim 3-4 "$ and a possible misalignment between the LABOCA and Spitzer maps of the order of $5^{\prime \prime}$. Together, this adds up to a search radius of $\sim 10^{\prime \prime}$.

Table 3 lists the coordinates of likely Spitzer counterparts, found using the software SExtractor (Bertin \& Arnouts 1996), and their measured flux densities in the 3.6, 4.5, 5.8, 8.0, and $24 \mu \mathrm{m}$ bands. We extracted sources with six adjacent pixels above $3.5 \sigma$, and used an aperture of five pixels for the photometry; applying the aperture corrections listed in the IRAC and MIPS data handbooks. The listed uncertainties on the flux densities are the statistical errors given by SExtractor. We estimate the systematic uncertainties to be $\sim 10 \%$. In the cases where a source has been extracted in one IRAC channel and not another we list upper limits.

We found in total 9 sources with infrared counterparts, and two where counterpart identification is complicated by the larger number of sources in the short wavelenght IRAC bands. For two sources we see an excess of flux in the MIPS map, but it is not significant enough to be extracted. Deeper MIPS imaging would therefore be useful. Deep high-resolution radio maps could also be used to distinguish between sources in the shorter wavelength bands. Comments about the individual sources are presented below. The lack of infrared counterparts was to be expected: for comparison, in the SHADES survey Pope et al. (2006) found 21 secure Spitzer counterparts to the 35 submm sources. An important result is that the large positional uncertainty of LABOCA $\left(\sim 10^{\prime \prime}\right.$ for most sources) is reduced because the pointing accuracy of Spitzer is less than $1^{\prime \prime}$.

Out of the nine sources with likely infrared counterparts, five have sufficient coverage to make it possible to investigate the shape of the mid-infrared SED. Ivison et al. (2004) suggested a diagram based on mid-infrared colors $S_{8.0} / S_{4.5}$ versus $S_{24} / S_{8.0}$. Based on the redshift tracks (the position as a function of redshift for a SED in color-color space) of typical starburst and AGN-type spectral energy distributions, such a diagram could distinguish between strong starburst SEDs and powerful AGN. A similar diagram was also used by Ivison et al. (2007) and Beelen et al. (2008), while Hainline et al. (2009) showed that its diagnostic capacity is limited. The five sources with identified counterparts in these three Spitzer bands have colors that lie in the starburst part of the diagram. This does not exclude the possibility of contributions from AGN to the dust heating, but indicates that the galaxies are starburst, rather than AGN, dominated. Infrared spectroscopic measurements could be used to investigate further the power source of those galaxies.

\subsection{Notes on individual sources}

Aside from Source \#1, none of the other sources have been detected previously in the mm or submm. Our observation of Source \#1 is discussed in the context of other observations. The few other sources for which complementary observations exist are discussed as well.

Source \#1: with a deboosted flux density of $48.0 \pm$ $1.3 \mathrm{mJy}$, this source is one of the brightest SMGs ever detected around $870 \mu \mathrm{m}$. This is very likely because of its proximity to a caustic line, which provides a large magnification. From their 

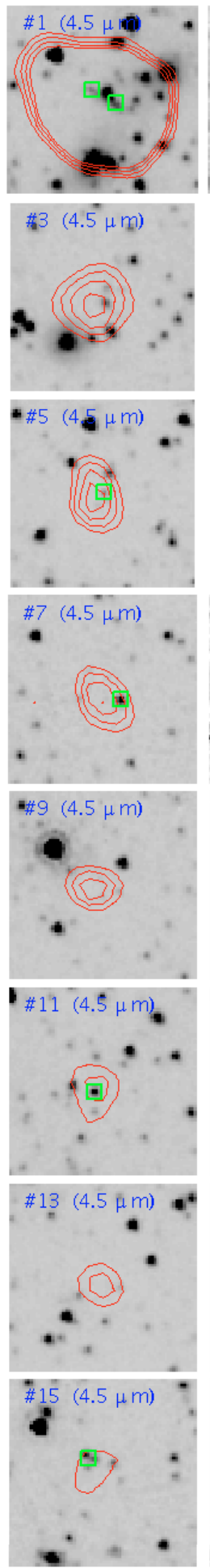
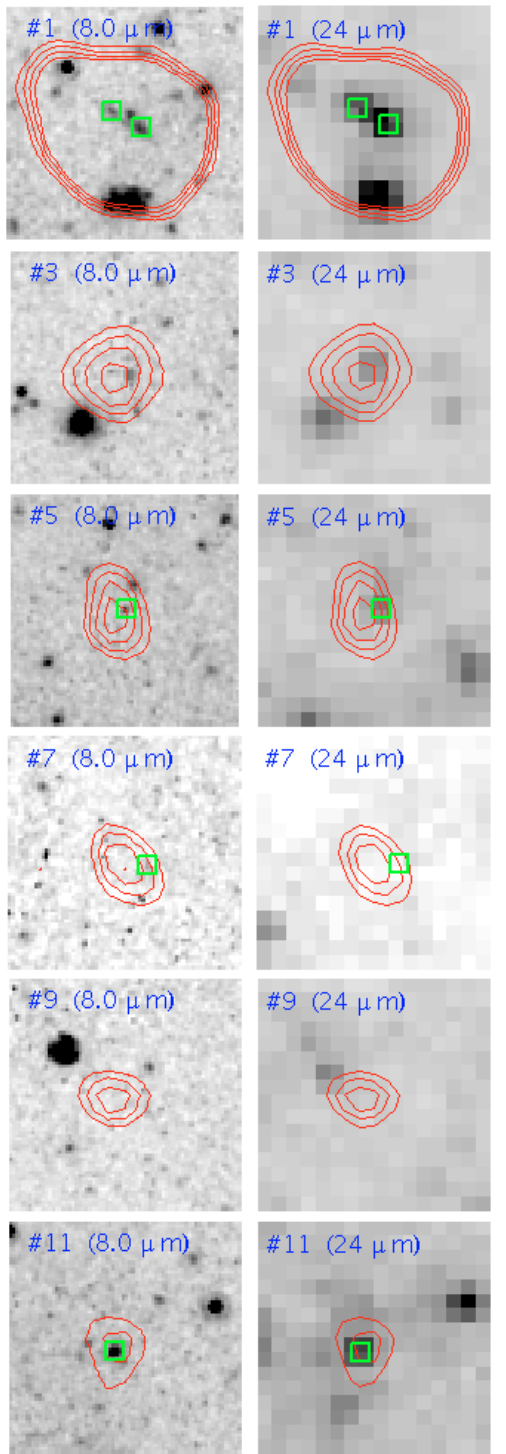

\#7 (24 $\mu \mathrm{m})$
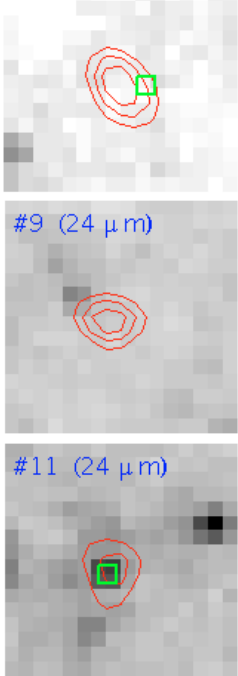

\#13 (24 $\mu \mathrm{m})$
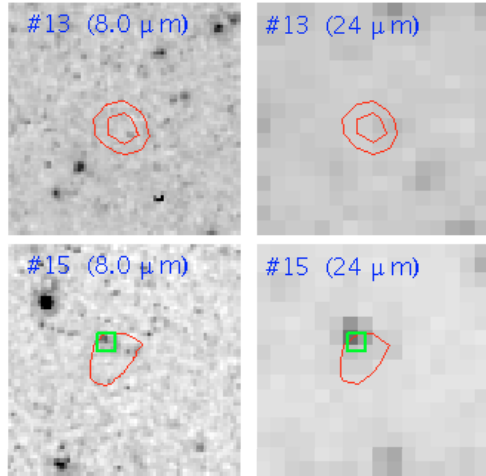
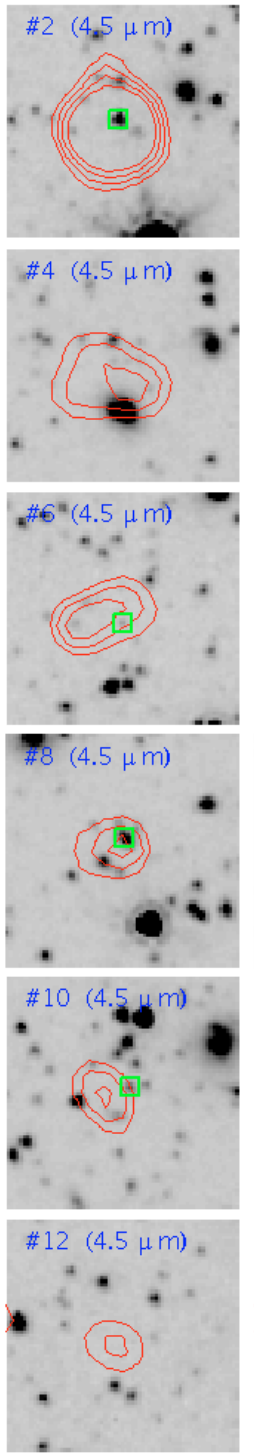

*14 (4.5 $\mathrm{m} \mathrm{m)}$
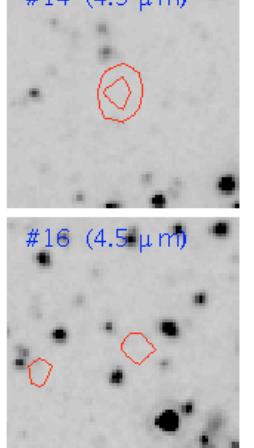
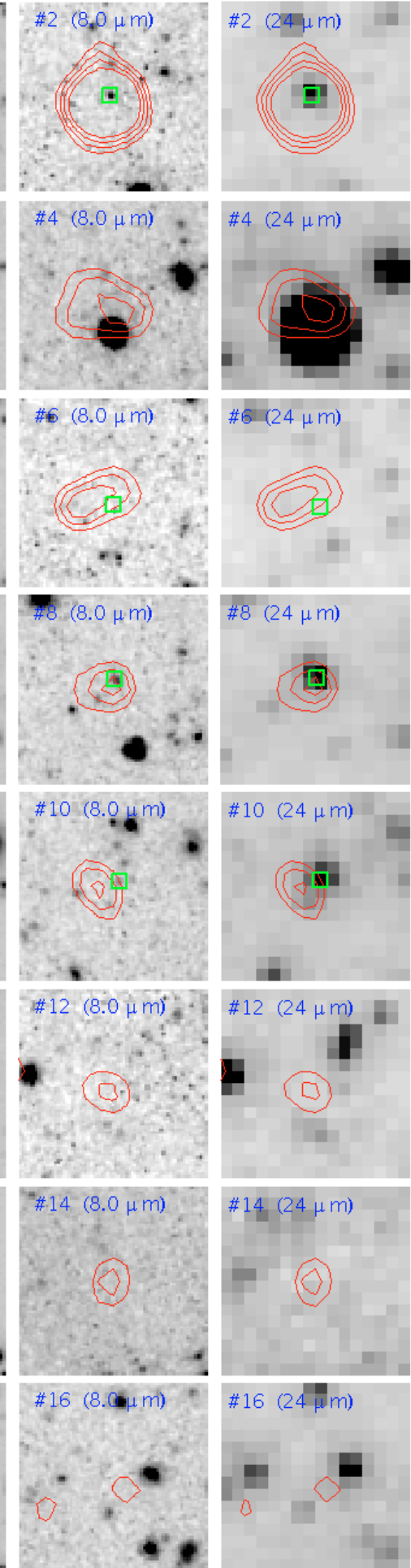

Fig. 8. $1^{\prime} \times 1^{\prime}$ cutouts of the Spitzer 4.5, 8.0 and $24 \mu \mathrm{m}$ maps centered on the positions of the LABOCA sources. The contours correspond to the 3.5, 4.5, 5.5 and $6.5 \sigma$-levels in the LABOCA map. The grey-scales for the Spitzer images are spaced between [0.0, 0.4], [2.4,2.8] and [20.25, 20.65] MJy/sr respectively and the pixel sizes are $0.85,0.85$ and 3.5 arcsec. We have also marked the positions of the likely infrared counterparts to the submm sources. The counterpart association is described in detail in Sects. 5.1 and 5.2.

lensing model, Gonzalez et al. (2009) estimated that the two brighter images of the galaxy, A and B, which are separated by $8.6^{\prime \prime}$, have a magnification of 25 and 50 , whereas the third image, C, located $47^{\prime \prime}$ away from the first one, would have a magnification four times lower than the first one. Our final map was smoothed to 22" and our fit to the Source \#1 gives a position between the images $\mathrm{A}$ and $\mathrm{B}$; the flux that we measure comes most likely from those two images. The total magnification is therefore 75 .

In the LABOCA study of the protocluster J2142-4423 by Beelen et al. (2008) the brightest source has a flux density of $21.1 \mathrm{mJy}$. The flux density at $850 \mu \mathrm{m}$, which is one of the SCUBA wavelengths, can be extrapolated using a submm spectral index of 2.7, giving a flux density $7 \%$ higher than at 
Table 3. Photometry of possible infrared counterparts to the LABOCA sources.

\begin{tabular}{lcccccccc}
\hline \hline Source & $\begin{array}{c}\alpha(J 2000) \\
(\mathrm{h}: \mathrm{m}: \mathrm{s})\end{array}$ & $\begin{array}{c}\delta(J 2000) \\
\left({ }^{\circ}::^{\prime \prime}\right)\end{array}$ & $\begin{array}{c}d_{\mathrm{LABOCA}}{ }^{a} \\
\left({ }^{\prime \prime}\right)\end{array}$ & $\begin{array}{c}S_{3.6 \mu \mathrm{m}} \\
(\mu \mathrm{Jy})\end{array}$ & $\begin{array}{c}S_{4.5 \mu \mathrm{m}} \\
(\mu \mathrm{Jy})\end{array}$ & $\begin{array}{c}S_{5.8 \mu \mathrm{m}} \\
(\mu \mathrm{Jy})\end{array}$ & $\begin{array}{c}S_{8.0 \mu \mathrm{m}} \\
(\mu \mathrm{Jy})\end{array}$ & $\begin{array}{c}S_{24 \mu \mathrm{m}} \\
(\mu \mathrm{Jy})\end{array}$ \\
\hline 2 & $06: 58: 24.59$ & $-55: 55: 12.2$ & 1.1 & $71.2 \pm 0.3$ & $99.5 \pm 0.5$ & $77.8 \pm 1.6$ & $53.1 \pm 1.9$ & $507.5 \pm 20.5$ \\
5 & $06: 58: 27.17$ & $-56: 01: 16.8$ & 1.0 & $12.1 \pm 0.2$ & $15.1 \pm 0.3$ & $<19.9$ & $24.9 \pm 1.5$ & $249.6 \pm 24.3$ \\
6 & $06: 58: 28.92$ & $-55: 53: 52.0$ & 3.6 & $13.7 \pm 0.4$ & $8.8 \pm 0.5$ & $<6.0$ & $<2.0$ & $<40$ \\
7 & $06: 59: 00.72$ & $-55: 52: 21.9$ & 6.8 & $89.2 \pm 0.2$ & $54.6 \pm 0.4$ & $26.8 \pm 1.5$ & $<18.7$ & $<40$ \\
8 & $06: 58: 23.96$ & $-55: 57: 19.5$ & 3.6 & $55.7 \pm 0.6$ & $68.5 \pm 0.6$ & $50.1 \pm 1.5$ & $40.5 \pm 1.5$ & $582.3 \pm 24.4$ \\
10 & $06: 58: 45.39$ & $-55: 58: 46.4$ & 2.3 & $17.5 \pm 0.7$ & $23.7 \pm 0.7$ & $28.2 \pm 1.9$ & $<18.4$ & $608.0 \pm 39.0$ \\
11 & $06: 58: 53.52$ & $-56: 00: 48.4$ & 4.3 & $33.4 \pm 0.4$ & $47.0 \pm 0.4$ & $72.2 \pm 2.2$ & $88.5 \pm 1.6$ & $228.8 \pm 33.5$ \\
15 & $06: 58: 34.14$ & $-55: 54: 37.2$ & 5.3 & $46.1 \pm 0.3$ & $37.2 \pm 0.4$ & $37.4 \pm 1.4$ & $30.1 \pm 2.1$ & $301.3 \pm 15.5$ \\
\hline
\end{tabular}

Notes. ${ }^{(a)}$ Distance between the IRAC 1 position and the central LABOCA position.

$870 \mu \mathrm{m}$. The SCUBA flux of Source \#1 would therefore be around $51 \mathrm{mJy}$. For comparison, the brightest source in the SHADES survey (Coppin et al. 2006) has $22 \mathrm{mJy}$ (and a signalto-noise ratio of 4.9). In the submm survey of massive clusters of galaxies, Knudsen et al. (2008) detected two bright sources towards Abell 478 and Abell 2204, with flux densities of 25.0 and $22.2 \mathrm{mJy}$ respectively.

Source \#1 was detected using the AzTEC bolometer array at $1.1 \mathrm{~mm}$ (W08); we can compare the astrometric position, angular size and integrated flux density with the values measured at $870 \mu \mathrm{m}$.

- Position: the distance between the estimated central position of the two sources is $4.1^{\prime \prime}$. This is well within the pointing error margins of the two telescopes.

- Source size: W08 reports a source size of $36 \pm 1.3^{\prime \prime} \times$ $32 \pm 1.2^{\prime \prime}$. To compare that with the LABOCA source, we smoothed our map to the AzTEC resolution of 30", and fitted a two-dimensional Gaussian. The fitted $F W H M$ s at this resolution are $36.2 \pm 1.3^{\prime \prime} \times 30.1 \pm 1.2^{\prime \prime}$, in good agreement with the value of W08.

- Flux density: after removal of the contribution from the Sunyaev-Zeldovich effect, the flux density of the source at $1.1 \mathrm{~mm}$ was estimated to $13.5 \mathrm{mJy}$ (Wilson et al. 2008). This value, however, is too low and is being revised to about 20 mJy (Wilson, priv. comm.), which would give a spectral index $\alpha_{1100}^{870}=3.7$ between the two measurements.

Far-infrared observations of this source by the BLAST experiment were recently published by Rex et al. (2009). BLAST observed at wavelengths 250,350 and $500 \mu \mathrm{m}$, with $F W H M$ beam sizes of 36, 42 and 60", respectively. High-significance detections were reported in all three bands; the flux measurements were contaminated by that of an elliptical galaxy, which is a spectroscopically confirmed member of the Bullet cluster and which had to be modeled and subtracted. After making correction for the SZ flux and color (due to the width of the BLAST filters), the flux densities in the three bands are $94 \pm 30,96 \pm 27$ and $110 \pm 21 \mathrm{mJy}$ for bands ranging from shorter to longer wavelengths.

Figure 9 shows the spectral energy distribution of Source \#1. The measured data points are from from Spitzer, BLAST, LABOCA and AzTEC. We compared the models of Lagache et al. (2003) of template starburst galaxies to those data points. We used a magnification of 75 , assuming that the flux comes from images A and B discussed by Gonzalez et al. (2009). We display the SED of a starburst galaxy at redshift 2.9, which is the redshift estimate of Rex et al. (2009), with a total luminosity of $10^{11.8} L_{\odot}$. The SED of a more redshifted galaxy, at redshift 3.9,

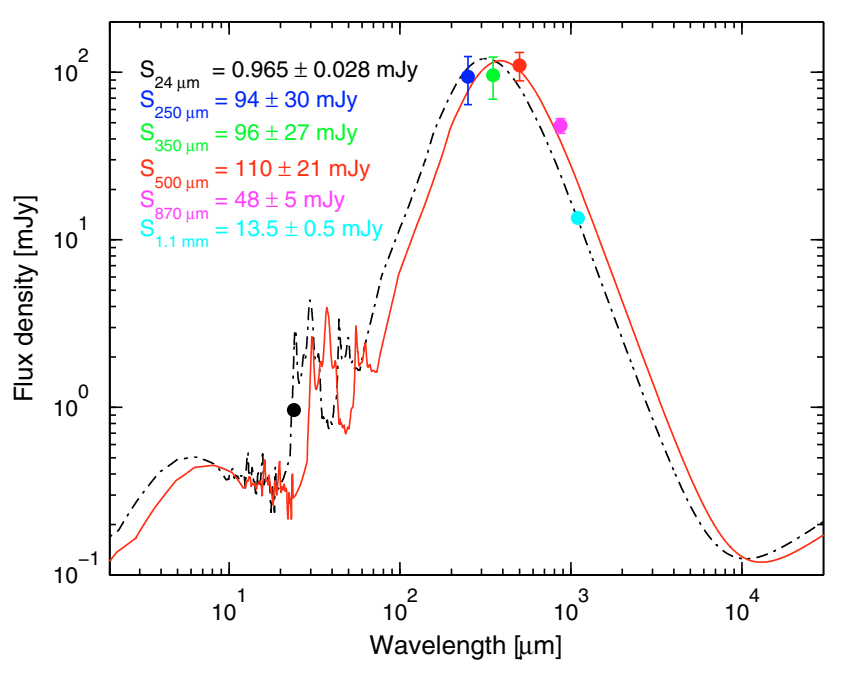

Fig. 9. Spectral energy distribution of Source \#1. The curves show modeled SEDs of starburst galaxies taken from the templates of Lagache et al. (2003) magnified by a factor of 75 . The magnification factor is taken from the lensing model used by Gonzalez et al. (2009) who find a magnification of 25 for the first image and of 50 for the second. The dotted-dashed curve corresponds to a starburst galaxy of intrinsic total luminosity $L_{\text {tot }}=10^{11.8} L_{\odot}$ redshifted to $z=2.9$. The solid curve shows the SED of a starburst galaxy of $L_{\mathrm{tot}}=10^{11.9} L_{\odot}$ redshifted to $z=3.9$. The data points are the $24 \mu \mathrm{m}$ Spitzer measurement of Gonzalez et al. (2009), the 250, 350 and $500 \mu \mathrm{m}$ BLAST measurements of Rex et al. (2009), our $870 \mu \mathrm{m}$ LABOCA point, and the AzTEC $1.1 \mathrm{~mm}$ measurement of W08.

with a luminosity of $10^{11.9} L_{\odot}$ is in better agreement with the long-wavelength data. However, it does not fit the $24 \mu \mathrm{m}$ value. Although we have not done a formal fit, it seems that the current data would require a different template SED. Alternatively, the uncertainties on the data points may have been seriously underestimated.

We do not list any Spitzer photometry of source \#1 because accurate measurement would require not only processing the raw data but also a careful subtraction of the foreground elliptical that lies between the two images of that source. Such a work has been performed by Gonzalez et al. (2009) and we used their quoted values in the analysis.

Source \#2: this is our second most significant detection at Gaussian filter value 29.9 or signal-to-noise ratio $S / N \simeq 15$. No previous mm or submm detection of this source has been reported, although in the map showing the detection of Source \#1 in W08 (their Fig. 1) there is an indication that they also detected this source. 
Figure 8 shows that this galaxy has likely counterparts in all available Spitzer bands. There is a source to the north of the center of the LABOCA detection in the 3.6 and $4.5 \mu \mathrm{m}$ maps that might be the reason for the apparent elongation of the red contour towards the north. However, this source is outside the $10^{\prime \prime}$ search radius and does not show up in the $24 \mu \mathrm{m}$ map.

Source \#3: a bright, extended Spitzer source is seen to the southeast of the LABOCA position. Its large angular size at $8.0 \mu \mathrm{m}$ and its distance from the submm position indicates that it is not the infrared counterpart. It is detected in the 2MASS catalog, but no redshift is indicated. Two sources are detected within the $10^{\prime \prime}$ circle. The source closest to the LABOCA position is identified in all IRAC band, whereas the other source is only found in IRAC1 and IRAC2. Both these sources are very faint due to this source confusion we choose to not list a counterpart. A flux excess is identified in the MIPS map, but it is not significant enough to be extracted by SExtractor.

Source \#4: the identification of a counterpart to the submm source is complicated by the bright foreground source seen in all five Spitzer bands. This source, at $z=0.097$, is also found in the 2MASS catalog. In radio observations with the ATCA array, Liang et al. (2000) detected a source at the position of the foreground galaxy. The flux density at $1.344 \mathrm{GHz}$ is $0.8 \pm 0.05 \mathrm{mJy}$ and drops towards higher frequencies. The source is not detected at 4.4 and $8.8 \mathrm{GHz}$.

Due to the positional offset, it is not very likely that the submm emission seen in the LABOCA map is caused by the $z=0.097$ galaxy south of the submm detection. There is a possibility that a small part of the total LABOCA flux density is coming from this foreground source, but not a substantial fraction. A faint source is detected to the north of the central position, but its position is slightly outside the $10^{\prime \prime}$ search radius.

Source \#5: two sources with detections in the IRAC 1, 2 and 4 bands are found close to the central position. A MIPS detection is associated with the centralmost source, which is situated only 1 " from the LABOCA position. The other source lies $6^{\prime \prime}$ away which point towards a counterpart association with the central source. High resolution radio imaging is needed to completely differentiate between these two sources, but we list the most central source here as a counterpart. Another source, to the south-east, is detected only in IRAC1, and is likely too faint to be a counterpart.

Source \#6: a source is detected in the IRAC 1 and 2 bands south of the LABOCA position. The brighter source north-west of the central position is further out than the search radius.

Source \#7: a significant source is detected in the IRAC 1, 2 and 3 bands west of the center of this source. Its SED is declining towards the longer wavelength IRAC bands.

Source \#8: a counterpart is identified in all Spitzer bands to the north of the LABOCA central position.

Source \#9: almost 10" from the central position lies a significant source, which is probably too far out and too bright to be the SMG counterpart. Closer to the central position two IRAC1 sources are extracted, but their similar flux levels and distance to the center makes the counterpart association ambiguous. We therefore choose to not list a counterpart for this SMG.

Source \#10: a bright MIPS source with counterparts in IRAC 1,2 and 3 is situated close to the LABOCA central position. This is a tentative counterpart to the LABOCA source.

Source \#11: a possible counterpart is detected in all the Spitzer bands.

Source \#15: the source to the northeast is identified as a counterpart and exists in all the Spitzer bands.

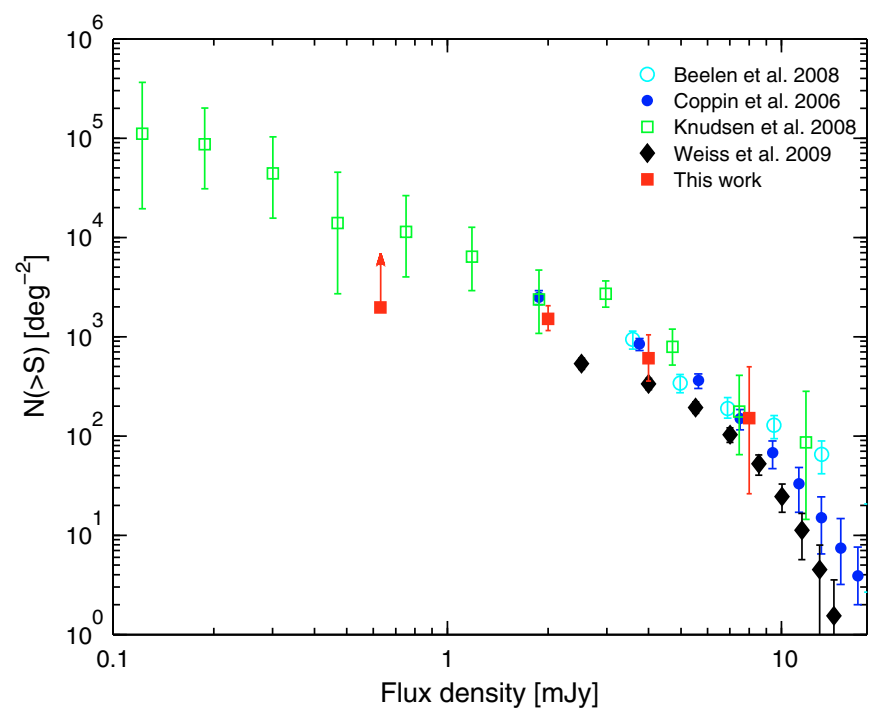

Fig. 10. Cumulative number counts derived from the central 10 arcmin of the $870 \mu \mathrm{m}$ map, after lensing and flux boosting correction. As the observations are highly complete, no completeness correction has been made (see the discussion in Sect. 4.3 and Fig. 6). For comparison, the resulting number counts from the LABOCA surveys of Weiß et al. (2009) and Beelen et al. (2008), and the SCUBA surveys of Coppin et al. (2006) and Knudsen et al. (2008) are displayed. The arrow on our point in the lowest bin indicates a lower limit: we reach the sub-mJy regime only because of the one bright source with an estimated magnification of 75. The surveyed area for this magnification is much smaller than the mean magnification factor of 3.3 described in the text, therefore this point only denotes a lower limit.

Source \#17: lies outside the $R=5^{\prime}$ circle from the center in a region when the noise is high. Our deboosting algorithm failed in that case, so we only quote the measured flux density of $23.6 \pm 5.9 \mathrm{mJy}$. This very bright source is also present in the AzTEC $1.1 \mathrm{~mm}$ map (Hughes \& Aretxaga, priv. comm.).

\subsection{Number counts}

Figure 10 shows the cumulative number counts, defined as the number density of sources with flux density larger than $S$, denoted $N(>S)$, derived from our observations and from other studies. There is good overall agreement between our results and those from previous LABOCA and SCUBA surveys, although our surveyed area is not as large as that of other studies, as reflected by the larger errorbars.

Because of the gravitational magnification, the surveyed area in the source plane is smaller than that in the image plane. We used the simple lensing model described in Sect. 4.5 to calculate the mean magnification in the central part of the map used for the analysis. We calculated a mean magnification factor of 3.3, i.e. the area of the source plane is 3.3 times smaller than the surveyed area in the image plane. The magnification of the source fluxes is also taken into account by using the de-magnified source flux densities listed in Table 2.

Our counts were inferred from the 13 sources found within the central 10 arcmin (the region within the red circle shown in Fig. 5), where the noise is uniform. From our 13 sources we construct the number counts in four flux-bins. We probe the counts down to $\sim 0.64 \mathrm{mJy}$, a regime which can be investigated only by utilizing foreground lensing clusters. The number of points per flux-bin and the counts are displayed in Table 4. We estimate 
Table 4. Cumulative number counts based on the central 10 arcminutes of our map.

\begin{tabular}{cccc}
\hline \hline $\begin{array}{c}S_{870} \\
\mathrm{mJy}\end{array}$ & $\begin{array}{c}N(>S) \\
\mathrm{deg}^{-2}\end{array}$ & $N_{\text {sources }}{ }^{a}$ & $N_{\text {per bin }}{ }^{b}$ \\
\hline 0.64 & $>1970$ & 13 & 3 \\
2.0 & $1510_{-360}^{+540}$ & 10 & 6 \\
4.0 & $610_{-250}^{+440}$ & 4 & 3 \\
8.0 & $150_{-130}^{+350}$ & 1 & 1 \\
\hline
\end{tabular}

Notes. ${ }^{(a)}$ Cumulative number of sources. ${ }^{(b)}$ The number of sources contributing to a bin (used to estimate the uncertainty).

errorbars for each data point from Poissonian statistics, using the tabulated values from Gehrels (1986).

In Fig. 10 we also show the results from the LESS survey (filled diamonds), which covered an area of $30^{\prime} \times 30^{\prime}$ of the Extended Chandra Deep Field South to a uniform noise level of $1.2 \mathrm{mJy} / \mathrm{beam}$ (Weiß et al. 2009). That survey has a noise level comparable to ours, but it covers an area which is 10 times larger. The apparent deficit of galaxies in the LESS has also been demonstrated in other wavebands (see Weiß et al. 2009 and references therein). The results of the LABOCA study of the protocluster J2142-4423 at $z=2.38$ (Beelen et al. 2008) are plotted in Fig. 10 as open circles. The excess seen at $S>3$ mJy might be an effect of clustering of submm sources in the protocluster.

We also compare with the SCUBA surveys from Coppin et al. (2006) (SHADES, filled circles) and Knudsen et al. (2008) (open boxes). The SHADES survey, which covered blank fields, probes the high-end of the number counts, while the Knudsen et al. survey targeted 10 lensing galaxy clusters and reached lower flux levels. Although our surveyed area is similar to that of Knudsen et al. (2008), we find only one sub-mJy source compared to the seven found by Knudsen et al. This is because their survey covers a larger area of high magnification.

\subsection{Resolving the cosmic infrared background}

It is interesting to investigate how much of the cosmic infrared background (CIB) flux is resolved in our LABOCA observation. The CIB was discovered by the COBE instruments FIRAS and DIRBE (Fixsen et al. 1998; Hauser et al. 1998). Those experiments detected an isotropic infrared background signal that was thought to originate from the integrated effect of star formation in the history of the universe (Dwek et al. 1998). But the coarse resolution of the COBE satellite made it impossible to resolve the sources responsible for the background signal. The detection of those sources had to wait for the invention of $\mathrm{mm}$ and submm bolometer cameras on large ground-based telescopes, resulting in angular resolutions of tens of arcseconds.

Because of the steep number counts, most of the flux coming from submm galaxies originates in low-flux sources. Therefore, observations of lensing foreground clusters that probe the faintest number counts potentially will resolve a large fraction of the CIB.

We use the central region of $10^{\prime}$ diameter for this calculation. The amount of CIB flux in that area, at $870 \mu \mathrm{m}$ is estimated from Eq. (1) of Dwek et al. (1998) and yields $\sim 900 \mathrm{mJy}$. The total flux in the sources extracted from the LABOCA map within that region (the same sources that are used for the number counts, but without the flux correction due to magnification) is $124 \pm 4.0 \mathrm{mJy}$. This calculation is valid because gravitational lensing preserves surface brightness. Thus, $14 \%$ of the CIB has been resolved in the LABOCA observations.

\subsection{Residual SZ emission}

Although the data have been filtered to remove the extended emission, some level of residual SZ emission may be present. Close inspection of the central region of the map shows extended emission between the two brighter sources, Source \#1 and \#2. We note that W08 (their Fig. 1) see a similar extended structure around the same central sources. In order to set an upper limit to the amount of residual SZ signal present in the region of the map covered by the Bullet cluster (which corresponds roughly to the area of a disk with an outer radius of about 2 arcmin), we calculated the difference between the excess flux density in that region (estimated from Fig. 4) and the flux density in resolved point sources in the same region. Removing the positive noise contribution estimated from the jackknife noise maps we find that $40 \mathrm{mJy}$ residual SZ emission might be present in the final map.

We can use the results of the APEX-SZ observations of the decrement at $150 \mathrm{GHz}$ by Halverson et al. (2009) to estimate the SZ flux density across the sky area covered by the Bullet cluster. Halverson et al. (2009) fitted an elliptical beta model using an X-ray prior on $\beta=1.04_{-0.10}^{+0.16}$ and found a core radius $\theta_{c}=142 \pm 18^{\prime \prime}$, an axial ratio of $0.89 \pm 0.07$ and a central Compton parameter $y_{0}=3.31 \pm 0.30 \times 10^{-4}$. Integrating the beta model over the central region with a maximum radius of 2 arcmin, and taking for simplicity a spherical model with the corresponding core radius, we estimate that the total SZ flux density from that area is of the order of $400 \mathrm{mJy}$. Therefore, we cannot exclude that a maximum of $10 \%$ of the total SZ flux of the Bullet cluster may remain in the map in the form of extended emission.

\section{Conclusions}

Continuum observations at $870 \mu \mathrm{m}$ of the Bullet cluster have been presented. The data were filtered to remove large-scale signal such as the Sunyaev-Zeldovich increment from the cluster. The main results are summarized below:

- Seventeen submm sources with signal-to-noise ratios larger than 4 were detected in the map. Their measured fluxes densities range from 4.6 to $48 \mathrm{mJy}$. For each source but one, which lies in a noisy area, we calculated the value of the flux density corrected for the flux boosting due to confusion noise and for the gravitational magnification by the cluster.

- The brightest submm source coincides with a previously reported galaxy at an estimated redshift of 2.7-2.9 detected by Spitzer and the AzTEC and BLAST experiments. With its flux density of $48 \mathrm{mJy}$ it is one of the brightest submm galaxy ever detected. After correction for gravitational magnification $|\mu|$, the intrinsic flux of the source is about $0.64(|\mu| / 75)^{-1} \mathrm{mJy}$.

- We found reliable infrared counterparts for nine of the submm sources. An infrared color-color analysis suggests that they have starburst-dominated spectral energy distributions.

- The cumulative number counts derived from the observations agree well with those from other surveys.

- The observations resolve $14 \%$ of the cosmic infrared background radiation at $870 \mu \mathrm{m}$, in a sky area of $\sim 78.5 \mathrm{arcmin}^{2}$. 

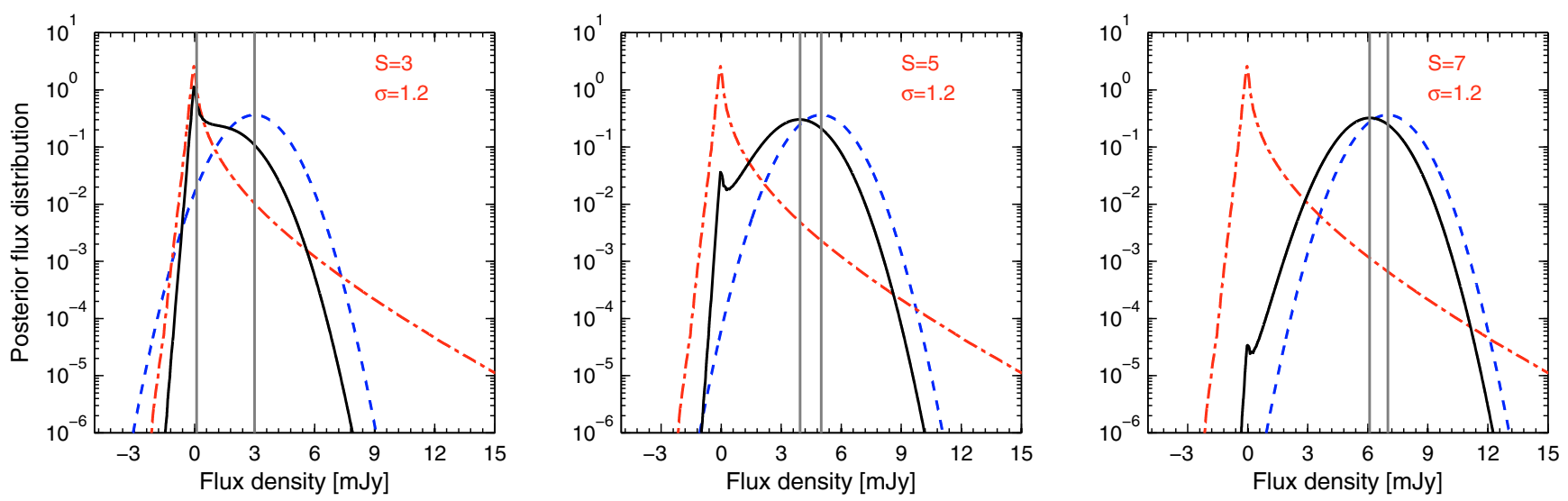

Fig. A.1. Solid curves: posterior flux distributions for measured flux densities of 3, 5 and $7 \mathrm{mJy}$ and a noise level of $1.2 \mathrm{mJy}$. Dashed curves: measured flux distributions, modelled as Gaussian functions. Dotted-dashed curve: prior flux density distributions estimated from noise-free simulations, populating maps with a Schechter flux distribution (Eq. (A.3)). The prior (red dotted-dashed) is identical in all three panels. The posterior flux distribution is the result of multiplying the blue dashed curve by the red dot-dashed curve and normalizing. The two vertical lines in each panel show the measured flux (rightmost line) and the maximum of the posterior flux distribution (leftmost line). The difference between the two lines indicates the amount of flux boosting.

We plan to apply a similar analysis to several other cluster fields for which we have LABOCA data. The results presented here will also be used to remove the contribution of the submm sources and obtain a map of the SZ increment at $870 \mu \mathrm{m}$ in the Bullet cluster with sub-arcminute angular resolution. Although the SZ decrement of the Bullet cluster has been observed using several instruments (e.g., SEST by Andreani et al. 1999, APEXSZ by Halverson et al. 2009, ACT by Hincks et al. 2009, and recently the South Pole Telescope by Plagge et al. 2009) the only observation of the increment was done using ACBAR at $275 \mathrm{GHz}$, with an angular resolution of 4.5' (Gomez et al. 2004). Removal of lensed background sources is not a trivial task, since it can also bias the SZ measurement (Loeb \& Refregier 1997). In the case of the Bullet cluster, the extremely bright lensed submm source close to the center of the cluster field has an integrated flux density comparable to that of the SZ flux from the central region of the cluster. A careful analysis must be performed to recover the SZ increment in such a system, and may require a joint analysis using measurements in other wavebands.

Acknowledgements. We thank the APEX staff for excellent support during the observations. C.H. and D.J. thank the Swedish Research Council for financial support. M.S., K.B., and F.B. acknowledge support from a grant within the DFG Priority Program 1177. H.Q. acknowledges partial support from the FONDAP Centro de Astrofísica. We thank Alexandre Beelen, John H. Black, David Hughes and Itziar Aretxaga for stimulating discussions, and Florian Pacaud for providing us with the XMM map of the Bullet cluster shown as contours in Fig. 7. We are grateful to Attila Kovács for writing Minicrush and being helpful to us during the data reduction. We thank the referee for a careful reading of the manuscript and useful comments.

This work is based in part on observations made with the Spitzer Space Telescope, which is operated by the Jet Propulsion Laboratory, California Institute of Technology under a contract with NASA.

\section{Appendix A: Flux deboosting}

This appendix describes the flux deboosting algorithm used in this work, following the prescription in Scott et al. (2008, Sect. 5.1), whose notation we use in the rest of this section.

The analysis builds upon Bayes theorem. It gives, for a source with a measured flux density $S_{\mathrm{m}}$ and uncertainty $\sigma_{\mathrm{m}}$, the posterior probability distribution of its intrinsic flux density $S_{\mathrm{i}}$ :

$P\left(S_{\mathrm{i}} \mid S_{\mathrm{m}}, \sigma_{\mathrm{m}}\right)=\frac{P\left(S_{\mathrm{i}}\right) P\left(S_{\mathrm{m}}, \sigma_{\mathrm{m}} \mid S_{\mathrm{i}}\right)}{P\left(S_{\mathrm{m}}, \sigma_{\mathrm{m}}\right)}$, where $P\left(S_{\mathrm{i}}\right)$ is the probability distribution of intrinsic flux densities, $P\left(S_{\mathrm{m}}, \sigma_{\mathrm{m}} \mid S_{\mathrm{i}}\right)$ is the likelihood of the observed data, and $P\left(S_{\mathrm{m}}, \sigma_{\mathrm{m}}\right)$ is a normalization.

The likelihood of observing the data, $P\left(S_{\mathrm{m}}, \sigma_{\mathrm{m}} \mid S_{\mathrm{i}}\right)$, is modeled by a Gaussian distribution. This is valid because of the Gaussian distribution of pixel values in the jackknife maps (see Fig. 4). The likelihood is

$P\left(S_{\mathrm{m}}, \sigma_{\mathrm{m}} \mid S_{\mathrm{i}}\right)=\frac{1}{\sqrt{2 \pi \sigma_{\mathrm{m}}^{2}}} \exp \left(-\frac{\left(S_{\mathrm{m}}-S_{\mathrm{i}}\right)^{2}}{2 \sigma_{\mathrm{m}}^{2}}\right)$.

We calculated the prior sky distribution $P\left(S_{\mathrm{i}}\right)$ from Monte Carlo simulations. We used a Schechter (1976) form of the number counts

$\frac{\mathrm{d} N}{\mathrm{~d} S}=N^{\prime}\left(\frac{S}{S^{\prime}}\right)^{\alpha+1} \exp \left(-S / S^{\prime}\right)$

as fitted to the source counts in the SCUBA SHADES survey of Coppin et al. (2006). We used $N^{\prime}=1703 \mathrm{deg}^{-2} \mathrm{mJy}^{-1}$, $S^{\prime}=3.1 \mathrm{mJy}$ and $\alpha=-2.0$, which have been scaled from the Coppin et al. values using a spectral index of 2.7. We then drew randomly source fluxes from that distribution and placed point sources (convolved with the beam) on noiseless sky maps. The position of each source was drawn from a uniform distribution, so no clustering was introduced in the simulation. We simulated a region of $10^{\prime} \times 10^{\prime}$. In order to reduce edge effects, each simulated map was $3^{\prime}$ larger, and a smaller map was extracted.

We generated $\sim 10^{6}$ sky maps and measured the pixel distribution in each of them. The mean distribution of pixel values in these maps is a measure of $P\left(S_{\mathrm{i}}\right)$.

From those simulated sky maps, we could calculate the posterior flux distribution of a source extracted from the map with flux density $S_{\mathrm{m}}$ and uncertainty $\sigma_{\mathrm{m}}$ : it is obtained by multiplying the prior $P\left(S_{\mathrm{i}}\right)$ (from the simulations) with the Gaussian distribution (Eq. (A.2)), and dividing by $P\left(S_{\mathrm{m}}, \sigma_{\mathrm{m}}\right)$.

Figure A. 1 shows examples of posterior flux distributions for three combinations of $S_{\mathrm{m}}$ and $\sigma_{\mathrm{m}}$. The amount of flux boosting for a certain source depends both on the signal-to-noise ratio and the value of the flux density; for example, for a signal-tonoise ratio of 4 , a source with a measured flux density of $10 \mathrm{mJy}$ will have been boosted more than a source with a flux density of $5 \mathrm{mJy}$. 


\section{References}

Andreani, P., Böhringer, H., dall'Oglio, G., et al. 1999, ApJ, 513, 23

Austermann, J. E., Dunlop, J. S., Perera, T. A., et al. 2010, MNRAS, 401, 160, 4 Beelen, A., Omont, A., Bavouzet, N., et al. 2008, A\&A, 485, 645

Bertin, E., \& Arnouts, S. 1996, A\&AS, 117, 393

Bertoldi, F., Carilli, C., Aravena, M., et al. 2007, ApJS, 172, 132

Blain, A. W. 1997, MNRAS, 290, 553

Blain, A. W. 1998, MNRAS, 297, 502

Blain, A. W., \& Longair, M. S. 1993, MNRAS, 264, 509

Blain, A. W., Smail, I., Ivison, R. J., Kneib, J.-P., \& Frayer, D. T. 2002, Phys. Rep., 369, 111

Borys, C., Chapman, S., Halpern, M., et al. 2003, MNRAS, 344, 385

Bradač, M., Clowe, D., Gonzalez, A. H., et al. 2006, ApJ, 652, 937

Chapman, S. C., Scott, D., Borys, C., et al. 2002, MNRAS, 330, 92

Clowe, D., Bradač, M., Gonzalez, A. H., et al. 2006, ApJ, 648, L109

Condon, J. J. 1974, ApJ, 188, 279

Coppin, K., Halpern, M., Scott, D., Borys, C., \& Chapman, S. 2005, MNRAS, 357,1022

Coppin, K., Chapin, E. L., Mortier, A. M. J., et al. 2006, MNRAS, 372, 1621

Cowie, L. L., Barger, A. J., \& Kneib, J.-P. 2002, AJ, 123, 2197

Dwek, E., Arendt, R. G., Hauser, M. G., et al. 1998, ApJ, 508, 106

Fazio, G. G., Hora, J. L., Allen, L. E., et al. 2004, ApJS, 154, 10

Fixsen, D. J., Dwek, E., Mather, J. C., Bennett, C. L., \& Shafer, R. A. 1998, ApJ, 508, 123

Gehrels, N. 1986, ApJ, 303, 336

Gomez, P., Romer, A. K., Peterson, J. B., et al. 2004, in Plasmas in the Laboratory and in the Universe: New Insights and New Challenges, ed. G. Bertin, D. Farina, \& R. Pozzoli, AIP Conf. Proc., 703, 361 Gonzalez, A. H., Clowe, D., Bradač, M., et al. 2009, ApJ, 691, 525 Güsten, R., Nyman, L. Å., Schilke, P., et al. 2006, A\&A, 454, L13 Hainline, L. J., Blain, A. W., Smail, I., et al. 2009, ApJ, 699, 1610 Halverson, N. W., Lanting, T., Ade, P. A. R., et al. 2009, ApJ, 701, 42
Hauser, M. G., Arendt, R. G., Kelsall, T., et al. 1998, ApJ, 508, 25 Hincks, A. D., Acquaviva, V., Ade, P., et al. 2009, [arXiv:0907.0461] Hogg, D. W., \& Turner, E. L. 1998, PASP, 110, 727

Ivison, R. J., Greve, T. R., Serjeant, S., et al. 2004, ApJS, 154, 124 Ivison, R. J., Greve, T. R., Dunlop, J. S., et al. 2007, MNRAS, 380, 199 Knudsen, K. K., van der Werf, P. P., \& Kneib, J.-P. 2008, MNRAS, 384, 1611 Kovács, A. 2008, in SPIE Conf., 7020 Lagache, G., Dole, H., \& Puget, J. 2003, MNRAS, 338, 555

Liang, H., Hunstead, R. W., Birkinshaw, M., et al. 2000, ApJ, 544, 686 Loeb, A., \& Refregier, A. 1997, ApJ, 476, L59+

Markevitch, M., Gonzalez, A. H., David, L., et al. 2002, ApJ, 567, L27

Nord, M., Basu, K., Pacaud, F., et al. 2009, A\&A, 506, 623

Plagge, T., Benson, B. A., Ade, P. A. R., et al. 2009, [arXiv: 0911.2444]

Pope, A., Scott, D., Dickinson, M., et al. 2006, MNRAS, 370, 1185

Rex, M., Ade, P. A. R., Aretxaga, I., et al. 2009, ApJ, 703, 348

Rieke, G. H., Young, E. T., Engelbracht, C. W., et al. 2004, ApJS, 154, 25

Sanders, D. B., \& Mirabel, I. F. 1996, ARA\&A, 34, 749

Schechter, P. 1976, ApJ, 203, 297

Schneider, P., \& Weiss, A. 1986, A\&A, 164, 237

Schneider, P., Ehlers, J., \& Falco, E. E. 1992, Gravitational Lenses, ed. E. J. F. E. E. Schneider, P.

Scott, S. E., Dunlop, J. S., \& Serjeant, S. 2006, MNRAS, 370, 1057

Scott, K. S., Austermann, J. E., Perera, T. A., et al. 2008, MNRAS, 385, 2225

Serjeant, S., Dunlop, J. S., Mann, R. G., et al. 2003, MNRAS, 344, 887

Shin, E. M., \& Evans, N. W. 2008, MNRAS, 390, 505

Siringo, G., Kreysa, E., Kovács, A., et al. 2009, A\&A, 497, 945

Smail, I., Ivison, R. J., \& Blain, A. W. 1997, ApJ, 490, L5+

Smail, I., Ivison, R. J., Blain, A. W., et al. 1998, ApJ, 507, L21

Smail, I., Ivison, R. J., Owen, F. N., Blain, A. W., \& Kneib, J.-P. 2000, ApJ, 528, 612

Springel, V., \& Farrar, G. R. 2007, MNRAS, 380, 911

Weiß, A., Kovács, A., Coppin, K., et al. 2009, ApJ, 707, 1201

Wilson, G. W., Hughes, D. H., Aretxaga, I., et al. 2008, MNRAS, 390, 1061 